\title{
Functional $\alpha 6 \beta 4$ acetylcholine receptor expression enables pharmacological testing of nicotinic agonists with analgesic properties
}

\author{
Daniel Knowland, Shenyan Gu, William A. Eckert III, G. Brent Dawe, Jose A. Matta, James Limberis, Alan D. Wickenden, \\ Anindya Bhattacharya, and David S. Bredt
}

Neuroscience Discovery, Janssen Pharmaceutical Companies of Johnson \& Johnson, San Diego, California, USA.

\begin{abstract}
The $\alpha 6 \beta 4$ nicotinic acetylcholine receptor ( $\mathrm{nAChR}$ ) is enriched in dorsal root ganglia neurons and is an attractive non-opioid therapeutic target for pain. However, difficulty expressing human $\alpha 6 \beta 4$ receptors in recombinant systems has precluded drug discovery. Here, genome-wide screening identified accessory proteins that enable reconstitution of human $\alpha 6 \beta 4 \mathrm{nAChRs.}$ BARP, an auxiliary subunit of voltage-dependent calcium channels, promoted $\alpha 6 \beta 4$ surface expression while IRE1 $\alpha$, an unfolded protein response sensor, enhanced $\alpha 6 \beta 4$ receptor assembly. Effects on $\alpha 6 \beta 4$ involve BARP's $\mathrm{N}$-terminal region and IRE1a's splicing of XBP1 mRNA. Furthermore, clinical efficacy of nicotinic agents in relieving neuropathic pain best correlated with their activity on $\alpha 6 \beta 4$. Finally, BARP-knockout, but not NACHO-knockout mice lacked nicotine-induced antiallodynia, highlighting the functional importance of $\alpha 6 \beta 4$ in pain. These results identify roles for IRE1 $\alpha$ and BARP in neurotransmitter receptor assembly and unlock drug discovery for the previously elusive $\alpha 6 \beta 4$ receptor.
\end{abstract}

\section{Introduction}

Chronic pain is a serious unmet public health issue in need of non-opioid analgesics. Epibatidine has long been recognized as a potent analgesic through actions on nicotinic acetylcholine receptors (nAChRs), yet negative side effects preclude its clinical use. nAChRs transduce synaptic signals and mediate diverse physiological actions of ACh in the brain and periphery (1-6). nAChRs comprise a large family of homo- and heteropentamers assembled from $10 \alpha(\alpha 1-\alpha 10, \alpha 8$ only found in chick) and $4 \beta$ ( $\beta 1-$ $\beta 4)$ subunits, each with discrete expression patterns. The $\alpha 4 \beta 2$ receptor is thought to be the subtype mediating nAChR-induced analgesia; however, despite significant investment there are no approved nicotinic agents for analgesia, suggesting that a different subtype may be involved (7).

Recently, animal and human studies have implicated dorsal root ganglia-enriched (DRG-enriched) $\alpha 6 \beta 4$ receptors in chronic pain states. Phenotypic analysis of inbred mice found that $\alpha 6$ levels in DRGs vary inversely with injury-induced allodynia, and loss-offunction variants in the $\alpha 6$ promoter region correlate with chronic postoperative pain (8). Furthermore, $\alpha 6$-knockout ( $\alpha 6-\mathrm{KO})$ mice suffer increased allodynia, whereas $\alpha 6$ gain-of-function models show attenuated pain levels (8). Collectively, $\alpha 6$-containing recep-

\section{Related Commentary: p. 5685}

Authorship note: SG and WAE contributed equally to this work.

Conflict of interest: The authors are all full-time employees of Janssen Pharmaceutical Companies of Johnson and Johnson.

Copyright: (5) 2020, American Society for Clinical Investigation.

Submitted: May 15, 2020; Accepted: August 6, 2020; Published: October 19, 2020

Reference information: J Clin Invest. 2020;130(11):6158-6170.

https://doi.org/10.1172/JCl140311. tors in the DRG represent an attractive non-opioid approach for the treatment of pain.

Although nAChRs are appealing therapeutic targets, drug discovery and basic science efforts are hampered because most subtypes are not expressed as functional proteins in cell lines. Especially problematic are human $\alpha 6$-containing receptors, which do not form functional channels in any recombinant expression system $(9,10)$. We previously identified NACHO, an endoplasmic reticulum (ER) protein, which serves as a clientspecific chaperone that promotes function of many nAChRs and is essential for assembly of $\alpha 7$ homopentamers $(11,12)$. NACHO also mediates assembly of dopamine neuron-enriched $\alpha 6 \beta 2 \beta 3$ receptors, which also require additional protein accessories including $\beta$-anchoring and -regulatory protein (BARP), SULT2B1, and LAMP5 (13). Whether DRG-enriched $\alpha 6 \beta 4$ shows similar dependency on NACHO and other accessory components for functional expression is unknown.

To assess this, we performed genome-wide cDNA screening for proteins that enable robust functional expression of human $\alpha 6 \beta 4$ receptors. Our screen identified BARP and inositolrequiring enzyme-1 $\alpha$ (IRE1 $\alpha$ ) as $\alpha 6 \beta 4$ enhancers. BARP is a single-pass transmembrane protein that was first identified in association with voltage-gated calcium channel $\beta$ subunits (14) and was later found as an accessory component of $\alpha 6 \beta 2 \beta 3$ nAChRs (13), while IRE1 $\alpha$ is a constituent of the unfolded protein response (UPR) (15). We found that the efficacy of nicotinic agonists to treat neuropathic pain best correlates with their activity on $\alpha 6 \beta 4$ receptors. Furthermore, BARP-KO mice, which show diminished $\alpha 6 \beta 4$ surface expression in DRG, have blunted antiallodynic responses to nicotine and ABT-594, a clinically effective nicotinic agent, suggesting that $\alpha 6 \beta 4$ activity is critical for neuropathic pain relief. 
A

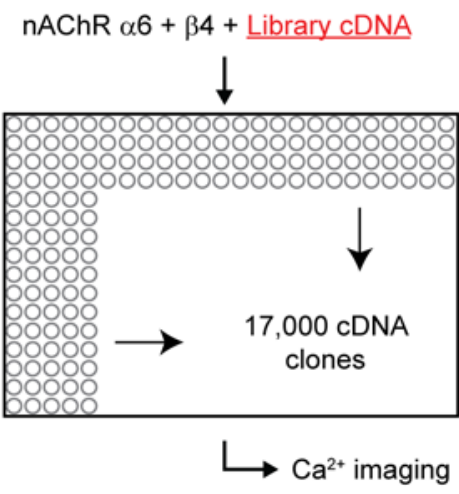

B

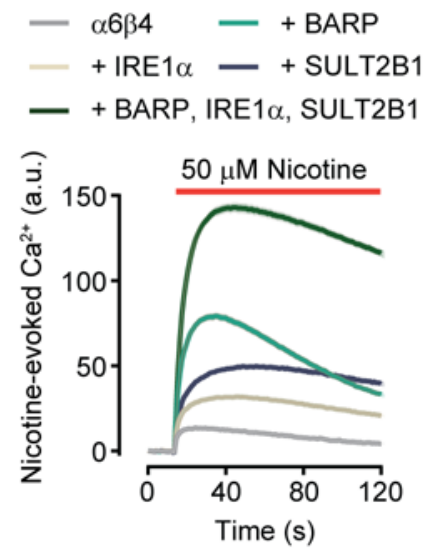

C

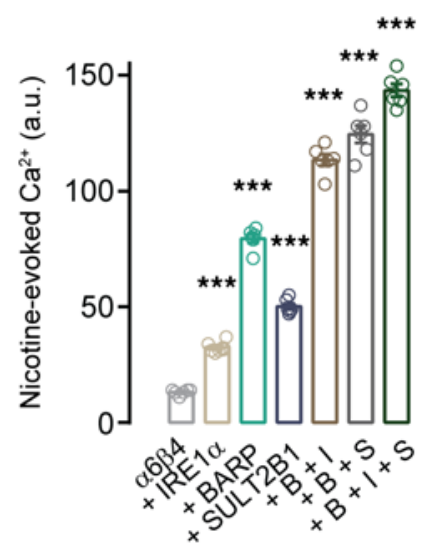

D

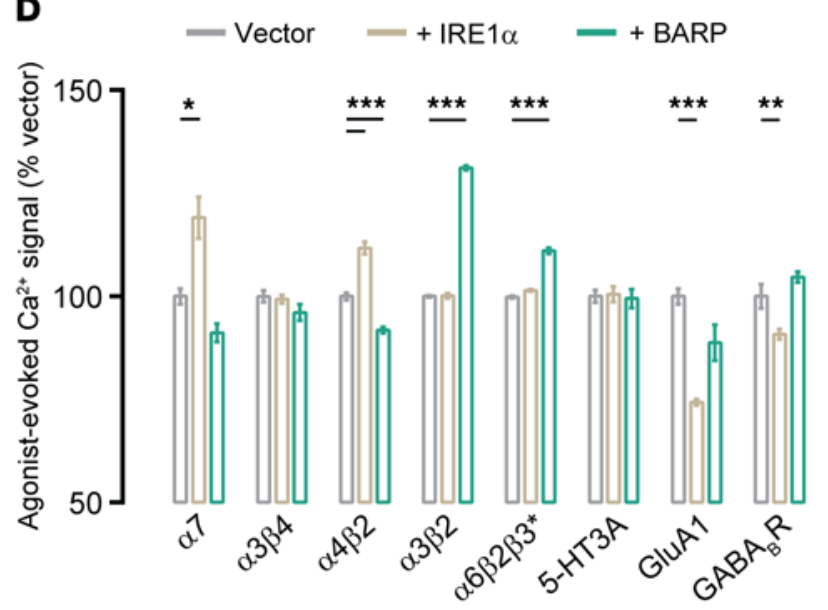

E $\alpha 6 \beta 4+\operatorname{IRE} 1 \alpha$
$\mathbf{F}$

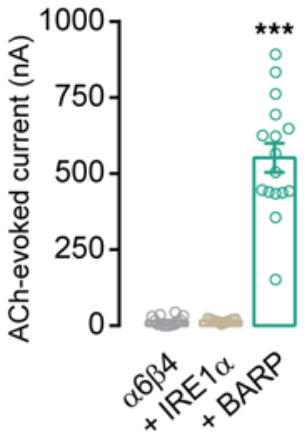

Figure 1. Genomic screening identifies chaperones for functional $\mathbf{\alpha 6 \beta 4}$ reconstitution. (A) Schematic of genomic screening. HEK293T cells were cotransfected with cDNAs encoding $\alpha 6$ and $\beta 4$ and individual plasmids from a genome-wide expression library. Ca ${ }^{2+}$ signals were measured in a fluorescence imaging plate reader (FLIPR). (B) Exemplary FLIPR traces showing nicotine-induced $\mathrm{Ca}^{2+}$ responses for indicated transfections. (C) Quantification of FLIPR signals. Activity of $\alpha 6 \beta 4$ is enhanced by BARP, IRE1 $\alpha$, and SULT2B1. $n=6$ for each group. B, BARP; I, IRE1 $\alpha$; S, SULT2B1. (D) Quantification of FLIPR $\mathrm{Ca}^{2+}$ response from other nAChRs and other ion channels (5-HT3A and GluA1), and $\mathrm{G}$ protein-coupled receptor (CABA $\mathrm{R}$ ) cotransfected with either BARP or IRE1 $\alpha$. nAChRs were stimulated with $50 \mu \mathrm{M}$ nicotine, 5-HT3A with $100 \mu \mathrm{M}$ serotonin, GluA1 with $100 \mu \mathrm{M}$ glutamate plus $100 \mu \mathrm{M}$ cyclothiazide, and $\mathrm{CABA}_{\mathrm{B}} \mathrm{R}$ with $100 \mu \mathrm{M}$ GABA. $n=6$ each. Responses normalized to that of vector-transfected cells $(100 \%)$. (E) Current traces from Xenopus oocytes injected with indicated cRNAs and stimulated with a 2-second pulse of $250 \mu \mathrm{M}$ ACh. (F) Quantification of current amplitude (absolute value) responses in E: $n=14$ 13 , and 16 oocytes for $\alpha 6 \beta 4, \alpha 6$ b4 plus IRE $1 \alpha$, and $\alpha 6 \beta 4$ plus BARP, respectively. ${ }^{*} P<0.05,{ }^{* *} P<0.01,{ }^{* * *} P<0.001$ by 1-way ANOVA with Dunnett's post hoc correction for multiple comparisons to vector (C, D, and F). C: $F_{6,35}=511.4$. D: $\alpha 7, F_{2,15}=18.08 ; \alpha 4 \beta 2, F_{2,15}=86.27 ; \alpha 3 \beta 2, F_{2,15}=1171 ; \alpha 6 \beta 2 \beta 3^{*}, F_{2,15}=143.9$; CluA1, $F_{2,15}=21.46 ; \mathrm{CABA}_{\mathrm{B}} \mathrm{R}, F_{2,15}=12.85$. F: $F_{2,40}=106.7$. Graphs are the mean \pm SEM and depict 1 experiment that was replicated with similar results.

\section{Results}

Genome-wide screening identifies BARP and IRE1 $\alpha$ as $\alpha 6 \beta 4$ accessory proteins. To identify functional regulators of $\alpha 6 \beta 4$, we cotransfected HEK293T cells with cDNAs encoding $\alpha 6$ and $\beta 4$ along with individual clones from a library of approximately 17,000 plasmids that express nearly all predicted human proteins. Using a fluorescence imaging plate reader (FLIPR) to measure nicotine-evoked $\mathrm{Ca}^{2+}$ responses, we identified 2 clones that augmented responses from $\alpha 6 \beta 4$ (Figure 1, A-C). The clone that most robustly enhanced the FLIPR signal was BARP, which we previously identified as an auxiliary subunit of $\alpha 6 \beta 2 \beta 3$ receptors (11). In those previous studies, we found that SULT2B1, LAMP5, and NACHO are also accessories for $\alpha 6 \beta 2 \beta 3$ (13). Here, we found that SULT2B1, but not NACHO or LAMP5, also augmented nicotine-evoked responses from $\alpha 6 \beta 4$ (Figure 1, B and C; Supplemental Figure 1A; supplemental material available online with this article; https://doi. org/10.1172/JCI140311DS1).

The second-best enhancer of $\alpha 6 \beta 4$ activity identified from the screen was IRE1 $\alpha$ of the UPR (15) (Figures 1, B and C). By contrast, 2 other UPR components, PERK and ATF6 (16), did not affect $\alpha 6 \beta 4$ FLIPR responses (Supplemental Figure 1A). We confirmed that these nicotine-evoked $\mathrm{Ca}^{2+}$ signals were mediated by $\alpha 6$, as $\alpha$-conotoxin MII, an $\alpha 6$-selective antagonist (17), completely blocked responses from $\alpha 6 \beta 4$, but not those from $\alpha 4 \beta 2$ or $\alpha 3 \beta 4$ (Supplemental Figure 1, B and C).

We next asked whether BARP and IRE1 $\alpha$ could also enhance responses from other nAChR subtypes. BARP promoted function of $\alpha 6 \beta 2 \beta 3^{*}$ (where ${ }^{*}$ indicates the use of the $\beta 3$ mutant sub- 
unit [V273S]) and $\alpha 3 \beta 2$ but had no effects on the other nAChRs tested (Figure 1D). Interestingly, IRE1 $\alpha$ enhanced $\alpha 7, \alpha 4 \beta 2$, and $\alpha 6 \beta 2 \beta 3^{*}$ to modest degrees (Figure 1D). Neither BARP nor IRE1 $\alpha$ increased responses from other ligand-gated ion channels including 5-HT3A or GluA1, or the class C GPCR GABA $_{B} R$ (Figure 1D).

We next explored the effects of BARP and IRE1 $\alpha$ using 2electrode voltage clamp in Xenopus oocytes. Previous studies have been unable to produce large, reliable human $\alpha 6 \beta 4$ currents in oocytes and instead have relied on chimeric or rodent receptors $(9$, 17-19). Similarly, we detected minimal to no ACh-evoked currents following oocyte injection with $\alpha 6$ and $\beta 4$ cRNAs (14.91 \pm 4.016 $\mathrm{nA})$. Strikingly, coinjection with BARP cRNA consistently enabled large ACh-evoked currents (551.9 $\pm 47.39 \mathrm{nA}$; Figure 1, E and F). By contrast, IRE1 $\alpha$ cRNA had no effect (15.78 \pm 1.549 nA; Figure 1, E and F). In whole-cell patch clamp electrophysiology on HEK293T cells transfected with $\alpha 6 \beta 4$ alone, small and inconsistent AChevoked $(1 \mathrm{mM})$ currents were evoked $(107.27 \pm 44.96 \mathrm{pA})$. However, similarly to oocytes, HEK293T cells coexpressing BARP with $\alpha 6 \beta 4$ produced larger, reliable currents $(566.06 \pm 226.02 \mathrm{pA})$, whereas those coexpressing IRE1 $\alpha$ did not $(20.30 \pm 10.43$ pA; Supplemental Figure 1, D and E). In HEK293T cells, BARP enhanced the peak amplitude of ACh-evoked currents from $\alpha 6 \beta 4$ but did not alter channel gating properties, as the steady-state-to-peak current ratio was the same in the absence or presence of BARP (Supplemental Figure 1F).

BARP and IRE1 $\alpha$ enable $\alpha 6 \beta 4$ functional expression through different mechanisms. We next explored cellular mechanisms underlying the effects of BARP and IRE1 $\alpha$ on $\alpha 6 \beta 4$. To assess $\alpha 6 \beta 4$ surface expression, we used a $\beta 4$ subunit containing a C-terminal, extracellular HA tag that does not interfere with receptor function (12). Cotransfection with BARP in HEK293T cells robustly enhanced $\alpha 6 \beta 4$ surface expression, whereas IRE1 $\alpha$ did not (Figure 2, A and B). This BARP effect was specific, as BARP did not enhance $\alpha 3 \beta 4$ surface expression (Figure 2, A and B). We additionally tagged $\alpha 6$ with an extracellular V5 epitope and again found that BARP, but not IRE1 $\alpha$, enhanced surface expression (Supplemental Figure 2, A and B). In alignment with functional data (Supplemental Figure $1 \mathrm{~A}$ ), we found that the $\alpha 6 \beta 2 \beta 3$ accessory protein SULT2B1 enhanced $\alpha 6 \beta 4$ surface expression, while NACHO and LAMP5 did not (Supplemental Figure 2, A and B).

BARP is a single-pass transmembrane protein with cytosolic motifs that bind voltage-gated calcium-channel (VGCC) $\beta$ subunits and negatively influences channel function (14). To determine whether BARP's effects on $\alpha 6 \beta 4$ and VGCCs share a common essential domain, we created a series of BARP mutants. BARP202, a truncated version lacking its VGCC $\beta$ subunit-binding domain, retained $\alpha 6 \beta 4$ functionality for both FLIPR and surface expression (Supplemental Figure 3). Further truncation of BARP's intracellular region (BARP101) or deletion of BARP's transmembrane region (BARP $\triangle \mathrm{TM}$ ) disrupted effects on $\alpha 6 \beta 4$ (Supplemental Figure 3). These experiments indicate that distinct domains are required for BARP's effects on $\alpha 6 \beta 4$ and VGCCs.

As IRE1 $\alpha$ did not enhance $\alpha 6 \beta 4$ surface expression, we assessed effects on receptor assembly. Orthosteric ligands such as $\left[{ }^{3} \mathrm{H}\right]$ epibatidine interact at the interface of assembled nAChR subunits, and their binding quantifies receptor oligomerization (20). We found that IRE1 $\alpha$, but not BARP, increased $\left[{ }^{3} \mathrm{H}\right]$ epibatidine binding to cotransfected $\alpha 6 \beta 4$ (Figure $2 \mathrm{C}$ ); however, neither BARP nor IRE1 $\alpha$ affected total $\alpha 6$ protein levels (Supplemental Figure 2C).

To assess protein interactions, we immunoprecipitated solubilized membranes from HEK293T cells cotransfected with $\alpha 6$ and $\beta 4$ along with accessory proteins. As expected, immunoprecipitation of V5-tagged $\alpha 6$ revealed association with $\beta 4$ (Figure 2D). We also observed an interaction with BARP and $\alpha 6 \beta 4$, indicating that BARP directly binds to $\alpha 6 \beta 4$. However, we observed no interaction with IRE1 $\alpha$ (Figure 2D). We confirmed the specificity of the immunoprecipitation, as BARP was absent from immunoprecipitation when cotransfected with only $\beta 4$ (Figure 2D, second lane). Taken together, our results demonstrate 2 separate roles for BARP and IRE1 $\alpha$ that work to augment $\alpha 6 \beta 4$ : IRE1 $\alpha$ enhances receptor assembly, while BARP promotes trafficking to the membrane by directly binding to $\alpha 6 \beta 4$.

$B A R P$ acts on the transmembrane and cytosolic regions of $\alpha 6$. We next investigated which regions of $\alpha 6$ are required for BARP effects. Because BARP regulates $\alpha 6 \beta 4$ but not $\alpha 4 \beta 4$, we constructed $\alpha 6 / \alpha 4$ receptor chimeras for cotransfection with $\beta 4$ (Figure $3 \mathrm{~A})$. BARP was inactive on a chimera containing the extracellular N-terminal region of $\alpha 6$ fused to the $\alpha 4$ transmembrane and intracellular regions ( $\alpha 6 \mathrm{~N} / 4$; Figure $3, \mathrm{~B}-\mathrm{D})$. Conversely, BARP robustly enhanced surface expression and FLIPR responses of the $\alpha 4 \mathrm{~N} / 6$ chimera (Figure 3, B-D). We further confirmed functionality in oocytes and again found that BARP enhanced AChevoked currents from $\alpha 4 \mathrm{~N} / 6$, but not from $\alpha 6 \mathrm{~N} / 4$ chimeras (Supplemental Figure 4, A and B). Taken together, these results show that the N-terminal extracellular domain of $\alpha 6$ is expendable for BARP-mediated effects, and that BARP directly interacts with $\alpha 6 \beta 4$ via its transmembrane regions.

RNase activity of IRE1 $\alpha$ is necessary and sufficient for $\alpha 6 \beta 4$ assembly. Because IRE1 $\alpha$ does not physically associate with $\alpha 6 \beta 4$, we wondered whether downstream signaling might be involved. Accumulation of unfolded proteins in the ER causes IRE1 $\alpha$ to dimerize and trans-autophosphorylate its kinase domain, which thereby activates its RNase domain to splice the transcription factor XBP1 to a unique protein, XBP1s (16). Accordingly, we generated IRE1 $\alpha$ mutants (Figure 4A) that abolish kinase (K599A, KINmut) or RNase (K907A, RNAmut) activities (21), as reflected by reduced XBP1s production (Figure $4 \mathrm{~B}$ ). Next, we cotransfected each mutant with BARP and measured FLIPR responses. Compared with WT IRE1 $\alpha$, both mutants reduced upregulation of nicotine-evoked $\mathrm{Ca}^{2+}$ responses from $\alpha 6 \beta 4$ (Figure $4 \mathrm{C}$ ). Additionally, STF-083010, an IRE1 $\alpha$ RNase inhibitor (22), blocked IRE1 $\alpha$-mediated enhancement of $\alpha 6 \beta 4$ function but had no effect on $\alpha 3 \beta 4$ (Supplemental Figure 5, B-D). In line with IRE1 $\alpha$ 's role in $\alpha 6 \beta 4$ assembly, IRE1 $\alpha$ KINmut and RNAmut reduced IRE1 $\alpha$ mediated enhancement of $\left[{ }^{3} \mathrm{H}\right]$ epibatidine binding (Figure 4D).

To isolate the contribution of IRE1 $\alpha$ 's RNase activity, we used a strategy developed by others (23) that combines an IRE1 $\alpha$ kinase-dead mutant (I642G, CONDmut) with a drug (1NM-PP1) that binds to the mutated kinase domain and initiates IRE1 $\alpha$ RNase activity independently of kinase activity (Figure 4A). Indeed, CONDmut showed reduced XBP1s, which was rescued with 1NM-PP1 (Figure 4B). In cells cotransfected with $\alpha 6 \beta 4$ and BARP, CONDmut IRE1 $\alpha$ reduced the nicotine-evoked FLIPR 
A

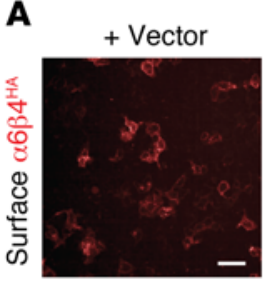

+ Vector

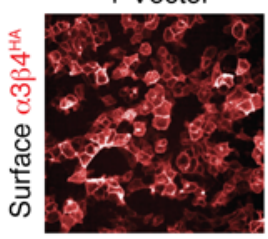

C

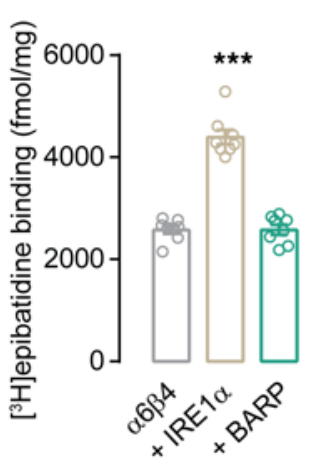

$+\operatorname{IRE} 1 \alpha$

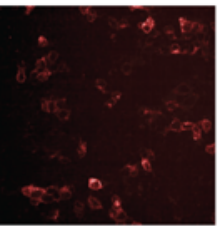

$+\operatorname{IRE} 1 \alpha$

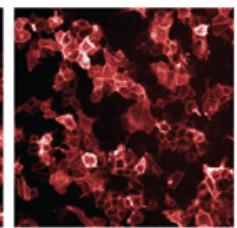

D
B

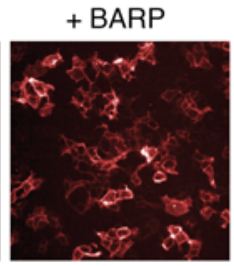

+ BARP

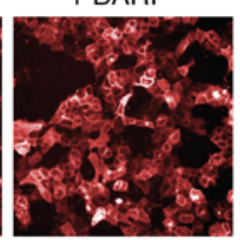

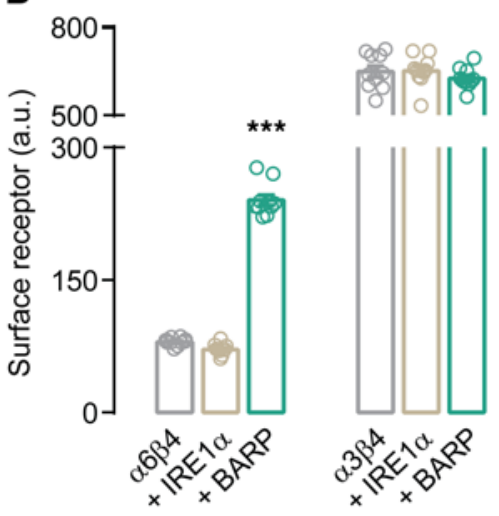
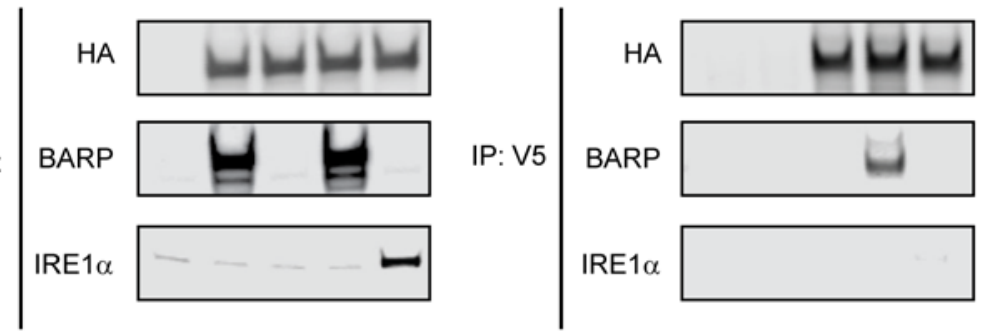

Figure 2. BARP and IRE1 $\alpha$ promote $\alpha \mathbf{6 \beta 4}$ function through distinct mechanisms. (A) Confocal images of $\alpha 6 \beta 4$ and $\alpha 3 \beta 4$ surface staining in transfected HEK293T cells. The $\beta 4$ subunit C-terminus contained an HA tag, which was visualized with an anti-HA antibody. (B) Quantification shows that BARP, but not IRE1 $\alpha$, enhances $\alpha 6 \beta 4$ surface staining. Neither has effects on $\alpha 3 \beta 4 . n=10$ for each group. Scale bar: $50 \mu \mathrm{m}$. (C) Quantification of [ ${ }^{3} \mathrm{H}$ ] $\mathrm{Cpibatidine} \mathrm{bind-}$ ing to HEK293T lysates transfected as indicated. $n=8$ for each condition. (D) Imm unoprecipitation with anti-V5 of solubilized HEK293T cells transfected as indicated. Immunoblotting shows that V5-tagged $\alpha 6$ associates with $\beta 4$ and BARP, but not with IRE1 $\alpha .{ }^{* *} P<0.001$ by 1 -way ANOVA with Dunnett's post hoc correction for multiple comparisons to vector (B and C). B: $F_{3,36}=515.9$. C: $F_{3,28}=106.7$. Graphs are the mean \pm SEM and depict 1 experiment that was replicated with similar results.

signal, which was restored with 1NM-PP1 (Figure 4E). Similarly, IRE1 $\alpha$ CONDmut reduced [ $\left.{ }^{3} \mathrm{H}\right]$ epibatidine binding to HEK293T cells transfected with $\alpha 6 \beta 4$ and this binding was also restored with 1NM-PP1 (Figure 4F). We next asked whether XBP1s itself can augment $\alpha 6 \beta 4$ (Supplemental Figure $5 \mathrm{~A}$ ). Indeed, transfection of XBP1s enhanced $\alpha 6 \beta 4$ assembly and function (Figure $4, G$ and $\mathrm{H})$. Collectively, these experiments suggest that IRE1 $\alpha$ RNase activity targeting $X B P 1$ is critical for $\alpha 6 \beta 4$ assembly.

Endogenous IRE1 $\alpha$ enhances $\alpha 6 \beta 4$ assembly. To assess whether activation of endogenous IRE1 $\alpha$ promotes $\alpha 6 \beta 4$ assembly, we used tunicamycin $(\mathrm{Tm})$, which inhibits $\mathrm{N}$-linked glycosylation, and thereby induces ER stress and stimulates the UPR $(24,25)$. Previous reports showed that Tm-induced IRE1 $\alpha$ activation peaks $4-8$ hours after treatment and reduces back to baseline by 24 hours (25). Similarly, we found that $100 \mathrm{ng} / \mathrm{mL} \mathrm{Tm}$ treatment for 4 , but not 12 or 24 hours stimulated IRE1 $\alpha$ activity to increase XBP1s (Figure 5A). Furthermore, $100 \mathrm{ng} / \mathrm{mL}$ Tm treatment for 4 , but not 12 or 24 hours enhanced $\alpha 6 \beta 4$ FLIPR responses (Figure 5, B and C) and [ $\left.{ }^{3} \mathrm{H}\right]$ epibatidine binding (Figure 5D) in $\alpha 6 \beta 4$-plus-BARP cotransfections.

To explore effects of IRE1 $\alpha$ activation on nAChRs in neurons, we first transduced cerebrocortical cultures with lentivi- ruses expressing $\alpha 6$ and $\beta 4$ subunits. Similarly to HEK293T cells, 4-hour, but not 24-hour, Tm treatment enhanced [ $\left.{ }^{3} \mathrm{H}\right]$ epibatidine binding (Figure 5E) and increased nicotine-evoked $\mathrm{Ca}^{2+}$ influx through $\alpha 6 \beta 4$ in neurons (Figure 5F). Furthermore, the IRE1 $\alpha$ RNase inhibitor STF-083010 blocked the Tm-induced increase in $\left[{ }^{3} \mathrm{H}\right]$ epibatidine binding (Figure $5 \mathrm{E}$ ). Also, Tm significantly enhanced nicotine-induced $\mathrm{Ca}^{2+}$ responses in untransduced cortical neurons (Figure $5 \mathrm{G}$ ), which express endogenous $\alpha 4 \beta 2$ receptors that are also regulated by IRE1 $\alpha$ (Figure 1D).

To confirm that Tm effects on $\alpha 6 \beta 4$ assembly specifically involve IRE1 $\alpha$ activation, we used CRISPR/Cas9 to disrupt IRE1 $\alpha$ in HEK293T cells. Immunoblotting demonstrated reduced or absent IRE1 $\alpha$ in IRE1 $\alpha$-heterozygous (IRE1 $\alpha$-HET) and IRE1 $\alpha$ KO cell lines, respectively (Figure $5 \mathrm{H}$ ). We also observed the expected reductions in Tm-induced XBP1s production in the IRE1 $\alpha$-HET and IRE1 $\alpha$-KO lines (Figure $5 \mathrm{H}$ ).

Interestingly, IRE1 $\alpha-\mathrm{KO}$ cells showed reduced nicotine-evoked $\alpha 6 \beta 4$-mediated FLIPR responses (Figure 5I). By contrast, $\alpha 3 \beta 4$ and 5HT3A responses increased in IRE1 $\alpha$-KO cells (Supplemental Figure 6). In line with FLIPR data, IRE1 $\alpha$-KO cells also exhibited reduced $\left[{ }^{3} \mathrm{H}\right]$ epibatidine binding (Figure $5 \mathrm{~J}$ ). As expected, 4-hour 
A
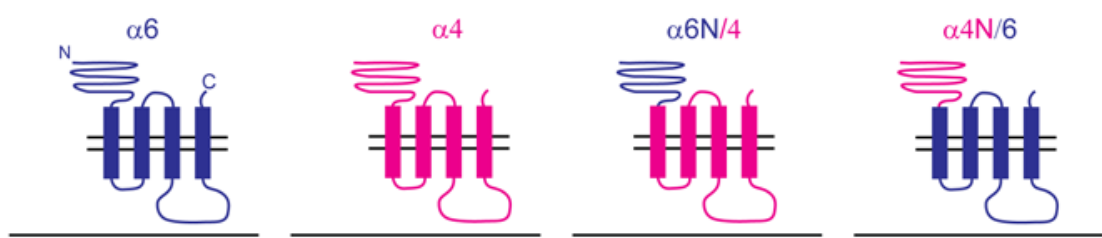

$\mathbf{B}$
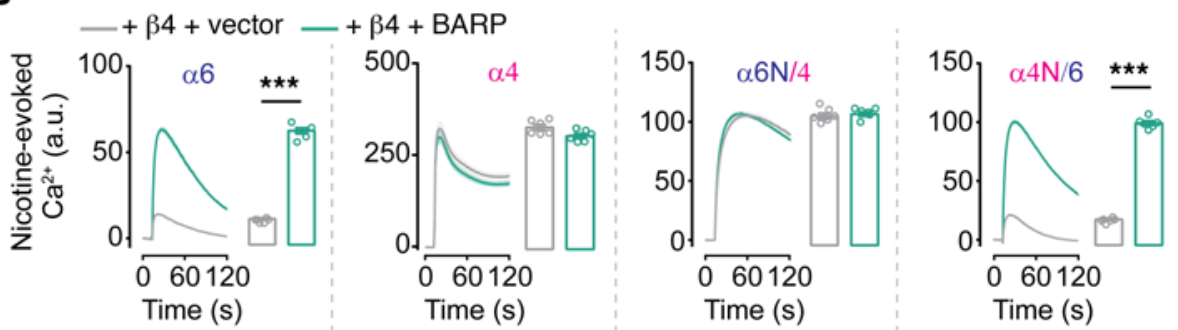

$\mathbf{C}$
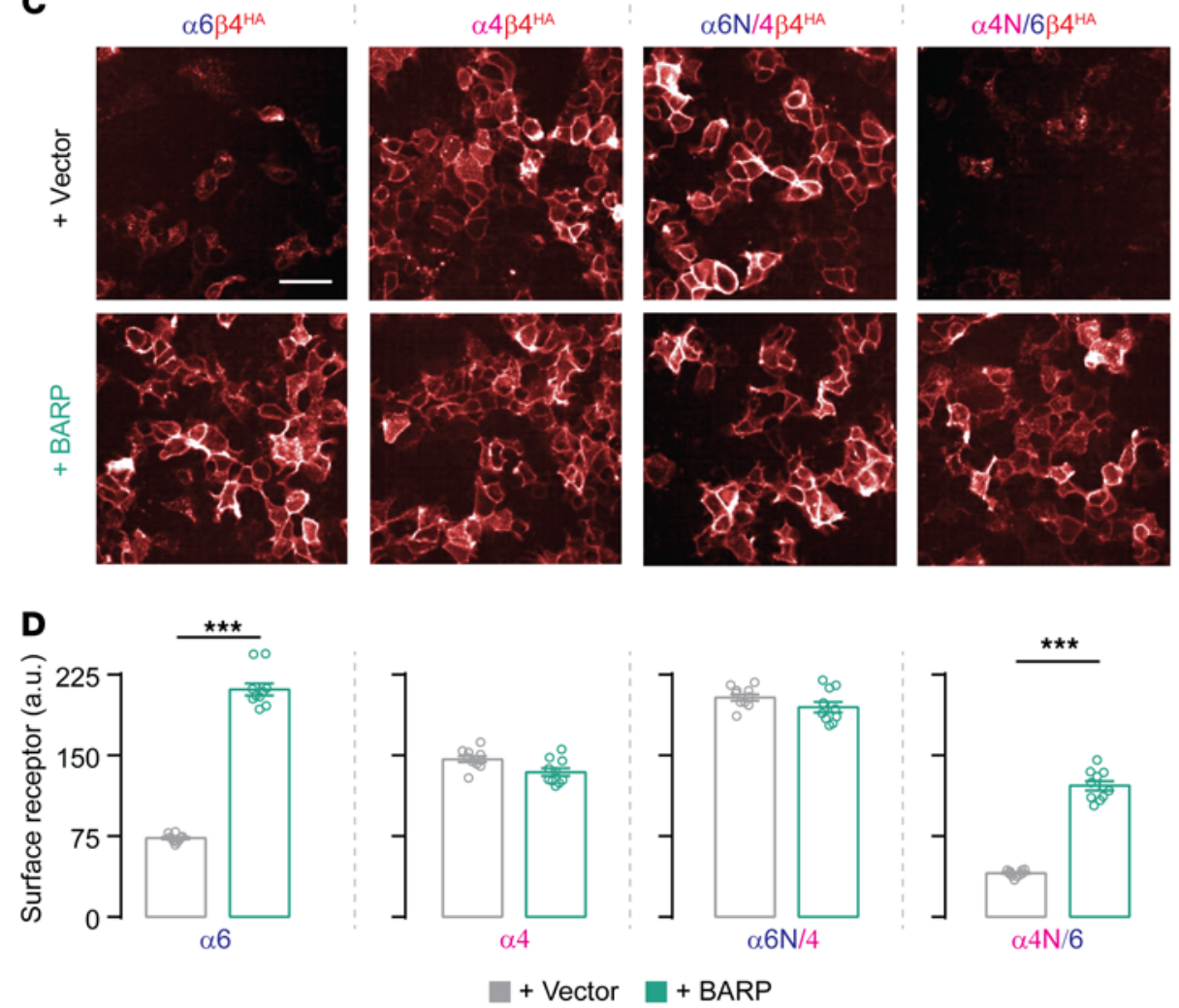

$\alpha 4 \mathrm{~N} / 6$
Figure 3. Transmembrane, but not $\mathrm{N}$-terminal region, of $\alpha \mathbf{6}$ is critical for BARP effect. (A) Schematics of $\alpha 6 / \alpha 4$ chimeras. (B) FLIPR traces and quantification of nicotine-evoked $(50 \mu \mathrm{M}) \mathrm{Ca}^{2+}$ responses. $n=6$ for each group. BARP enhances FLIPR responses only in $\alpha 6$ - and $\alpha 4 \mathrm{~N} / 6$-containing receptors cotransfected with $\beta 4$. (C) Representative confocal images for $\beta 4^{\mathrm{HA}}$ surface staining in cotransfected HEK293T cells with (bottom) and without (top) BARP. Scale bar: $50 \mu \mathrm{m}$. (D) Quantification of surface receptor intensity in C. $n=10$ for each group. All chimeric $\alpha 6 / \alpha 4$ nAChRs were cotransfected with $\beta 4$ into HEK293T cells. ${ }^{* * *} P$ $<0.0001$ by unpaired $t$ test (B and D). B: $\alpha 6 \beta 4, t=29.95 ; \alpha 4 \mathrm{~N} / 6, t=40.58$. D: $\alpha 6 \beta 4, t=24.04 ; \alpha 4 \mathrm{~N} / 6, t=$ 18.19. Graphs are the mean \pm SEM and depict 1 experiment that was replicated with similar results.

$B A R P$ regulates sensory neuron $\alpha 6 \beta 4$ and nicotine-induced antiallodynia. $\alpha 6$ and $\beta 4$ Subunit mRNAs are selectively coexpressed in DRG (Supplemental Figure 7A) (26). In addition, $\alpha 6 \beta 4$ mediated currents have been recorded in DRG neurons and $\alpha 6 \beta 4$ receptors there modulate pain responses $(8,27)$. Here, we detected a BARP-immunoreactive band in DRG and cerebral cortical protein extracts that comigrated with BARP from transfected HEK293T cells but was absent from BARP-KO tissues (Figure 6A). Because there are no suitable antibodies to detect endogenous $\alpha 6$ or $\beta 4$ (28), we transduced isolated DRG neurons with V5-tagged $\alpha 6$ and $\beta 4$ from WT and $B A R P-\mathrm{KO}$ mice. Compared with WT neurons, $B A R P-\mathrm{KO}$ neurons had reduced $\alpha 6 \beta 4$ surface expression but equivalent total $\alpha 6$ (Figure 6, B and C), showing that BARP promotes $\alpha 6 \beta 4$ surface expression in neurons. In contrast, $B A R P-\mathrm{KO}$ mice showed no difference from WT in $\alpha 4 \beta 2$ or endogenous $\alpha 7$ surface expression (Supplemental Figure 7, B-E).

The nicotinic agonist ABT-594, which is closely related to epibatidine, effectively treated diabetic neuropathic pain in clinical trials, but its development was discontinued due to side effects (29). A follow-up compound, ABT-894, developed to circumvent the nicotinic side effects, was not efficacious (30). The lack of efficacy for ABT-894 was hypothesized to reflect its increased specificity for $\alpha 4 \beta 2$ receptor, suggesting that other nAChRs underlie ABT-594's efficacy in neuropathic pain (30).

We wondered whether differential effects of ABT-894 and ABT-594 on $\alpha 6 \beta 4$ receptors could explain their contrasting clinical efficacies. We found that ABT-594, nicotine, and epibatidine - all of which have analgesic effects exhibited similar agonistic efficacies on $\alpha 4 \beta 2$ and $\alpha 6 \beta 4$ receptors (Figure 6, D and E; Supplemental Tables 1 and 2). In contrast, ABT-894 was only weakly efficacious at $\alpha 6 \beta 4$ as compared with $\alpha 4 \beta 2$ (Figure 6, D and E). All compounds Tm application increased $\left[{ }^{3} \mathrm{H}\right]$ epibatidine binding to levels comparable to transfected IRE1 $\alpha$ in WT cells (Figure $5 \mathrm{~K}$ ). In contrast, Tm failed to increase $\alpha 6 \beta 4\left[{ }^{3} \mathrm{H}\right]$ epibatidine binding in IRE1 $\alpha-\mathrm{KO}$ cells but could be rescued when transfected with exogenous IRE1 $\alpha$ (Figure 5K). These data demonstrate that endogenous IRE1 $\alpha$ activity supports $\alpha 6 \beta 4$ assembly and function. tested behaved as full agonists at $\alpha 3 \beta 4$. Together, these compounds' activities on $\alpha 6 \beta 4$ may provide an explanation for the clinical failure of ABT-894 in diabetic neuropathic pain.

To explore $\alpha 6 \beta 4$ 's role in neuropathic pain in vivo, we evaluated $B A R P-\mathrm{KO}$ animals in the spared nerve injury (SNI) model of neuropathic allodynia (31). No difference in mechanical allodynia 
A

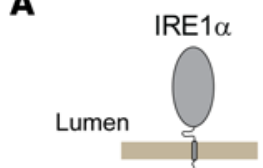

Kinase domain

RNase domain
K599A - Kinase mutant (KINmut) K907A - RNase mutant (RNAmut) I642G - Conditional mutant (CONDmut) $\rightarrow$ activated by 1 NM-PP1
B

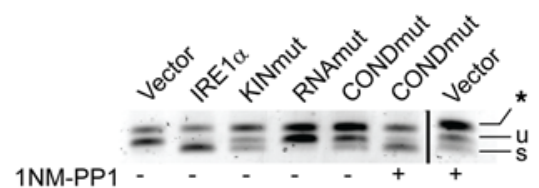

D

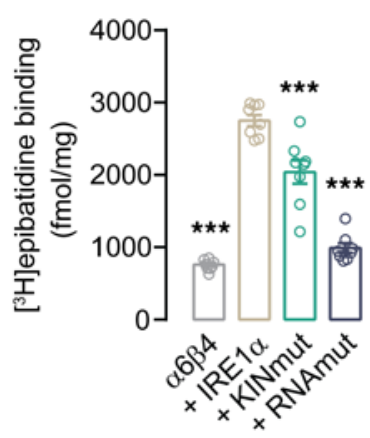

$\mathbf{F}$

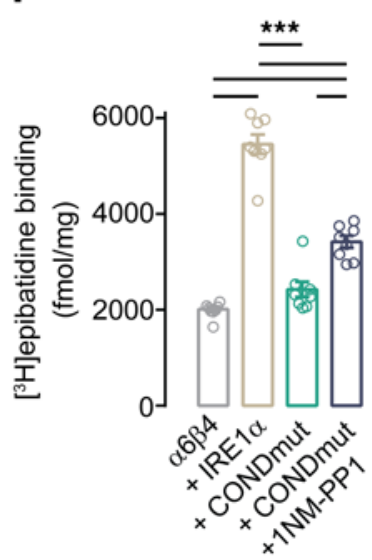

H

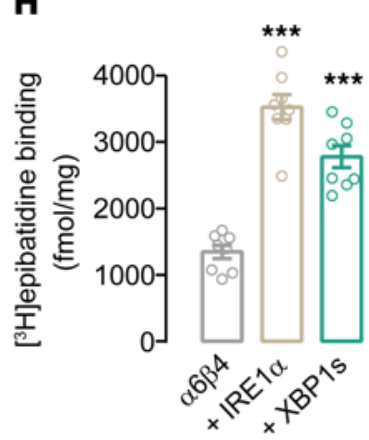

Figure 4. IRE1 $\alpha$ RNase activity and XBP1 splicing mediate assembly of $\boldsymbol{\alpha 6 \beta 4}$. (A) Schematics of IRE1 $\alpha$ kinase domain (K599A, KINmut), RNase domain (K907A, RNAmut), and conditional (I642G, CONDmut) mutants that were transfected into HEK293T cells. (B) RT-PCR shows that IRE1 $\alpha$ mutants decrease XBP1 splicing (lower band) as compared with WT IRE1 $\alpha$. 1NM-PP1 rescues XBP1s in I642G CONDmut. $u=$ fulllength unspliced XBP1, $s=$ spliced XBP1 (XBP1s), * = hybrid amplicon. (C) FLIPR traces (left) and quantification (right) of HEK293T cells transfected with WT IRE1 $\alpha$, K599A mutant, or K907A mutant. IRE1 $\alpha$ and mutants were cotransfected with BARP. $n=6$ for each group. (D) IRE1 $\alpha$ mutants reduce $\left[{ }^{3} \mathrm{H}\right]$ epibatidine binding in HEK293T cell lysates compared with WT IRE1 $\alpha . n=8$ for each condition. (E) FLIPR traces (left) and quantification (right) of $1642 \mathrm{C}$ CONDmut without and with $5 \mu \mathrm{M}$ 1NM-PP1, which rescued the FLIPR response. (F) CONDmut reduces $\left[{ }^{3} \mathrm{H}\right]$ epibatidine binding. RNase activation of CONDmut with $5 \mu \mathrm{M}$ 1NM-PP1 increases $\alpha 6 \beta 4$ assembly. $n=8$ for each condition. ( $\mathbf{G}$ and $\mathbf{H}$ ) FLIPR traces (G, left), quantification (G, right), and $\left[{ }^{3} \mathrm{H}\right]$ epibatidine binding $(\mathbf{H})$ from HEK293T cells cotransfected with $\alpha 6 \beta 4$ and XBP1s as indicated. $n=12$ for each condition in $\mathbf{G}, n=8$ for each group in $\mathbf{H} .{ }^{* *} P<0.001$ by 1 -way ANOVA with Dunnett's post hoc test to correct for multiple comparisons to WT IRE1 $\alpha$ (C and D), 1-way ANOVA with Tukey's multiple-comparisons post hoc test ( $\mathbf{E}$ and $\mathbf{F}$ ), unpaired $t$ test (G), or 1-way ANOVA with Dunnett's post hoc test to correct for multiple comparisons to vector (H). C: $F_{3,20}=702.6$. D: $F_{2,28}=92.92$. E $F_{3,20}=136 . \mathbf{F}: F_{3,28}=111.7 . \mathbf{G}: t=5.86$. H: $F_{2,21}=49.87$. Graphs are the mean \pm SEM and depict 1 experiment that was replicated with similar results. was measured before or up to 4 weeks after SNI surgery between WT and BARP-KOs $(0.250 \pm 0.066 \mathrm{~g}, 0.166 \pm 0.043 \mathrm{~g}$, respectively; Figure 6, F and G). Systemic nicotine administration exerts antiallodynia in rodent models of neuropathic pain $(8,32)$. Fitting with this, we found that intraperitoneal (i.p.) administration of nicotine $(1.0 \mathrm{mg} / \mathrm{kg})$ reduced mechanical allodynia following nerve injury in WT animals (Figure 6F). However, nicotine had no such relieving effect in BARP-KO mice (WT, $0.529 \pm 0.101 \mathrm{~g}$; BARP$\mathrm{KO}, 0.248 \pm 0.064 \mathrm{~g}$; Figure 6F).
We next asked whether the antiallodynic deficits observed in $B A R P-\mathrm{KO}$ mice were specific to $\alpha 6 \beta 4$. NACHO is an essential chaperone for brain $\alpha 7$ receptors and promotes assembly of other nAChRs such as $\alpha 4 \beta 2, \alpha 3 \beta 2$, and $\alpha 6 \beta 2 \beta 3$ (11). Further, NACHO knockouts show dramatically reduced brain epibatidine binding (12) and striatal $\alpha$-CTXMII binding (12). Importantly, we show here that $\alpha 6 \beta 4$ occupies a privileged role compared with other nAChR subtypes, as NACHO has no effect on $\alpha 6 \beta 4$ function (Supplemental Figure 1, A and E; Supplemental Figure 2, A and B). 

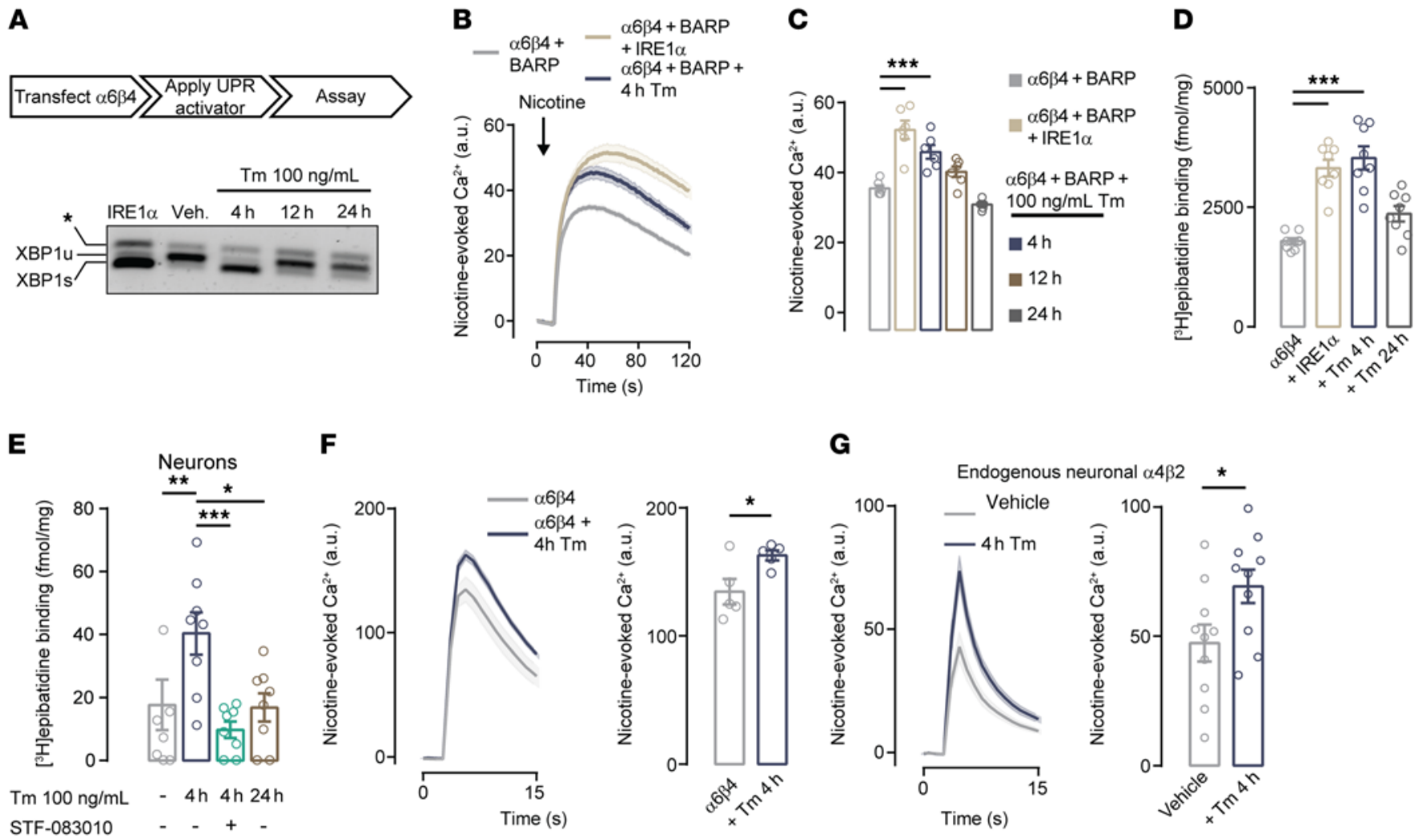

H

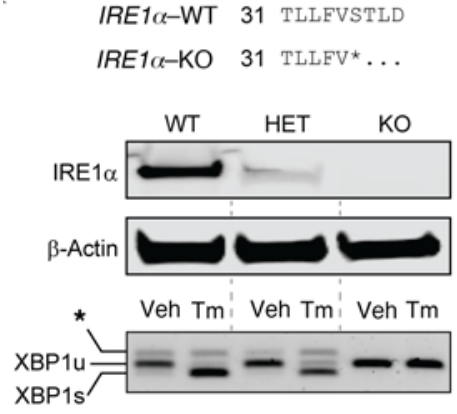

\section{I}

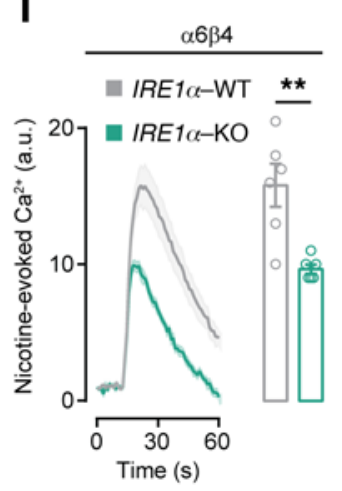

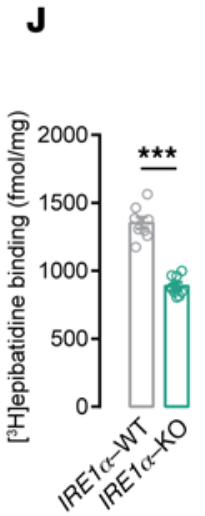

IRE $1 \alpha-\mathrm{WT}$

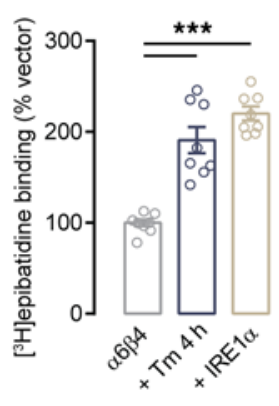

IRE $1 \alpha-\mathrm{KO}$

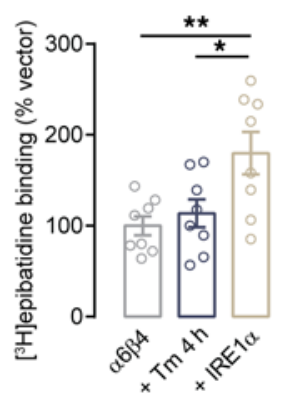

Figure 5. Endogenous IRE1 $\alpha$ enhances $\alpha \mathbf{6 \beta 4}$ assembly. (A) HEK293T cells were transfected with $\alpha 6 \beta 4$ and treated with tunicamycin (Tm, $100 \mathrm{ng} / \mathrm{mL}$ ) as indicated. Tm induced splicing of XBP1 (XBP1s) at 4 hours. XBP1u, unspliced XBP1. (B and C) Tm treatment for 4, but not 12 or 24 hours enhanced nicotine-induced $(7.5 \mu \mathrm{M}) \alpha 6 \beta 4$ FLIPR responses. $n=6$ for each condition except $n=5$ for BARP + IRE1 $\alpha$. (D) [ ${ }^{3} \mathrm{H}$ ] epibatidine binding to $\alpha 6 \beta 4$ in HEK293T cells with indicated treatments. $n=8$ each. $(E)$ [ $\left.{ }^{3} \mathrm{H}\right]$ epibatidine binding to cortical neuron lysates transduced with $\alpha 6 \beta 4$. IRE1 $\alpha$ inhibitor STF-083010 (20 $\mu \mathrm{M})$ applied 30 minutes before $\mathrm{Tm}(100 \mathrm{ng} / \mathrm{mL}) . n=8$ each. (F) Nicotine-evoked $(50 \mu \mathrm{M}) \mathrm{Ca}^{2+}$ responses in cortical neurons transduced with $\alpha 6 \beta 4$ lentiviral particles. $n=5$ for each condition. (C) Endogenous $\alpha 4 \beta 2$-mediated $\mathrm{Ca}^{2+}$ responses in cortical neurons. $n=10$ for each condition. (H) Top: CRISPR/Cas9mediated strategy for stop codon $\left(^{*}\right)$ insertion in IRE1 $\alpha$. Middle: Immunoblotting confirmed IRE1 $\alpha$ protein knockout. Bottom: IRE1 $\alpha$ activity in IRE1 $\alpha-$ heterozygous (IRE1 $\alpha$-HET) and IRE1 $\alpha$-knockout (IRE1 $\alpha$-KO) lines. RT-PCR shows that the KO line lacks XBP1 splicing activity. (I) Nicotine-evoked (100 $\mu$ M) FLIPR traces (left) and quantification (right) from IRE1 $\alpha$-WT and IRE1 $\alpha$-KO HEK293T cell lines transfected with $\alpha 6 \beta 4$ and BARP. $n=6$ for each condition. (J) [ ${ }^{3} \mathrm{H}$ ] epibatidine binding in IRE $1 \alpha-\mathrm{WT}$ and $I R E 1 \alpha-K O$ cells transfected with $\alpha 6 \beta 4 . n=8$ each. $(\mathrm{K})\left[{ }^{3} \mathrm{H}\right]$ epibatidine binding to cells transfected with $\alpha 6 \beta 4$. Tm treatment increased binding in WT, but not IRE1 $\alpha$-KO cells, and cotransfection with IRE1 $\alpha$ increased binding in both lines. $n=8$ each. ${ }^{*} P<0.05$; ${ }^{* *} P<$ $0.01 ;{ }^{* *} P<0.001$ by 1-way ANOVA with Dunnett's post hoc test compared with $\alpha 6 \beta 4$ alone (C and $\left.\mathbf{D}\right)$, unpaired $t$ test (F-J), or 1-way ANOVA with Tukey's post hoc test (E and K). C: $F_{4,25}=24.92$. D: $F_{3,28}=22.29$. E: $F_{3,27}=8.115$. K: $F_{2,21}=41.93$ for IRE1 $\alpha$-WT, $F_{2,21}=6.197$ for IRE1 $\alpha-K O$. Graphs are the mean \pm SEM and depict 1 experiment that was replicated with similar results.

To this end, we performed SNI surgery on NACHO-KO mice and tested mechanical allodynia. No difference in mechanical allodynia was observed after SNI surgery (Supplemental Figure 7F). Furthermore, NACHO-KO mice exhibited significant nicotine- induced antiallodynia at levels comparable to WT (gram threshold levels WT, $0.854 \pm 0.217 \mathrm{~g} ;$ NACHO-KO, $0.566 \pm 0.118 \mathrm{~g}$ ), indicating that the deficits seen in $B A R P-\mathrm{KO}$ mice were predominantly mediated via $\alpha 6 \beta 4$ (Supplemental Figure 7, F and G). 
A

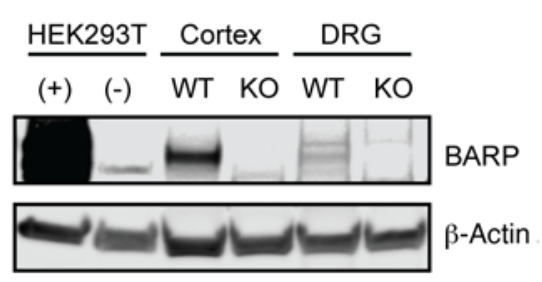

B

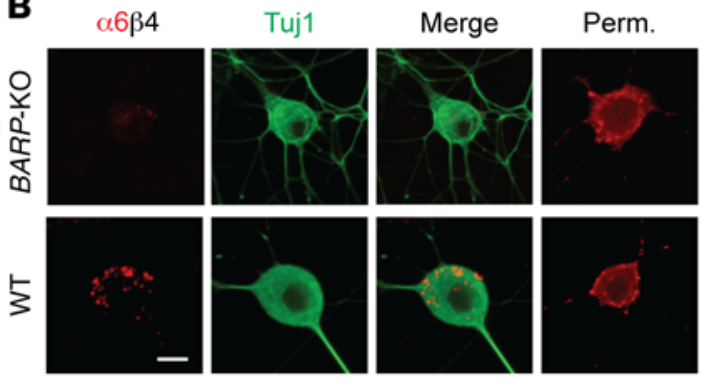

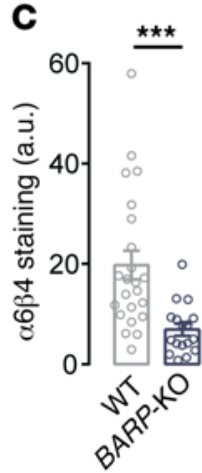
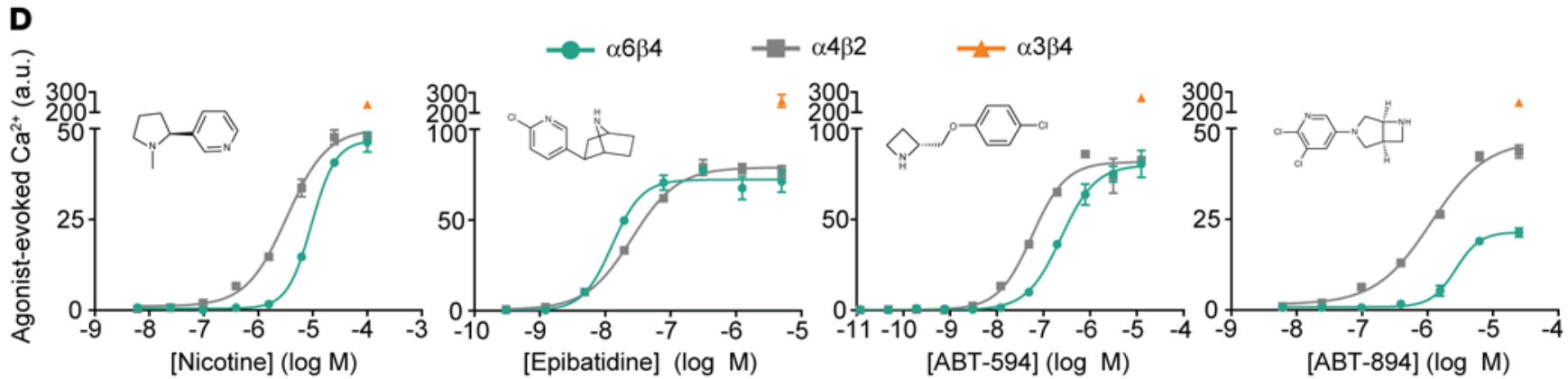

$\mathbf{E}$

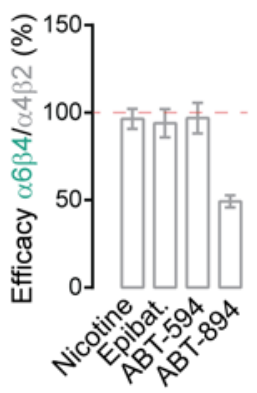

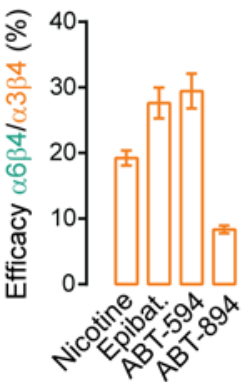
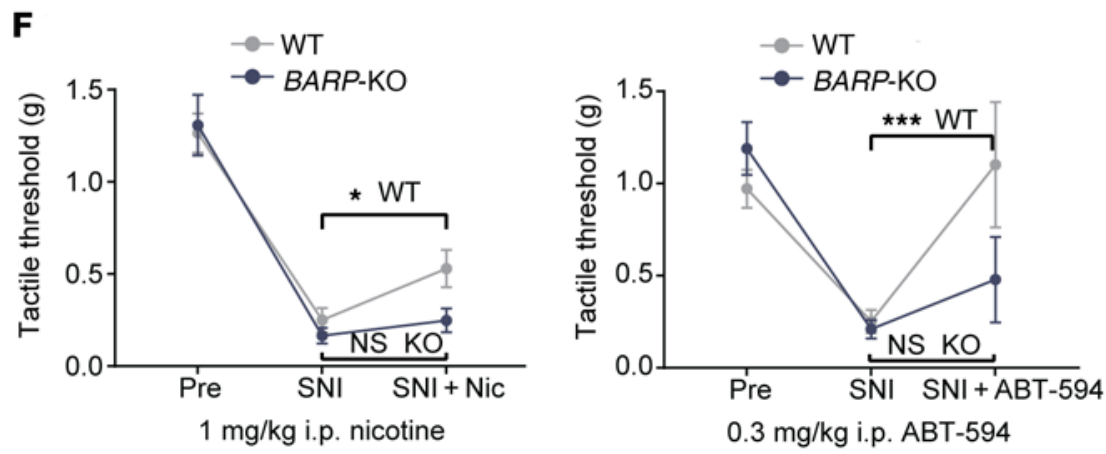

Figure 6. BARP promotes $\alpha \mathbf{6 \beta 4}$ function in vivo and mediates antiallodynia. (A) Immunoblotting identified BARP protein in cerebral cortex and dorsal root ganglia (DRG) from WT but not BARP-KO mice. BARP-transfected HEK293T cells and $\beta$-actin served as controls. (B and C) Imaging (B) and quantification (C) of surface $\alpha 6$ in DRG neurons from WT and BARP-KO mice. Neurons were transduced with lentivirus expressing $\alpha 6-V 5$ and $\beta 4$ subunits and were stained with anti-V5 antibody. Surface staining for $\alpha 6$ is reduced in BARP-KO neurons, whereas permeabilized (Perm) neurons show similar total levels of receptor. Scale bar: $10 \mu \mathrm{m} . n=23$ and 17 neurons for WT and BARP-KO, respectively. (D) FLIPR responses in transfected HEK293T cells stimulated with indicated compounds. Cells transfected with $\alpha 6 \beta 4$ were cotransfected with BARP, IRE1 $\alpha$, and SULT2B1 (for values see Supplemental Tables 1 and 2). Shown are concentration-response curves for $\alpha 6 \beta 4$ and $\alpha 4 \beta 2$ and a single high concentration for $\alpha 3 \beta 4$. (E) Quantification of maximal compound efficacy on $\alpha 6 \beta 4$ vs. $\alpha 4 \beta 2$ (left) or $\alpha 3 \beta 4$ (right). (F and $\mathbf{G}$ ) Mechanical allodynia was assessed in WT and BARP-KO mice before (Pre) and following spared nerve injury (SNI) surgery. WT, but not BARP-KO mice exhibited significant (F) nicotine-mediated and (G) ABT-594-mediated antiallodynia. $n=10$ and 12 for WT and BARP-KO, respectively in F. $n=11$ for each group for $\mathbf{C} .{ }^{*} P<0.05$; ${ }^{* *} P<0.001$ by Mann-Whitney test (C) or linear mixed-effects model for repeated measures comparing SNI-baseline to SNI-treatment time point (F and $\mathbf{G})$. C: $U=57$. F: $P=0.05$ for WT, SNI vs. SNI + nicotine; $P=0.35$ for $B A R P-K O$. G: $P<0.001$ for WT, SNI vs. SNI + nicotine; $P=0.4$ for BARP-KO. Graphs are the mean $\pm \mathrm{SEM}$. Graphs in C-E are representative of 1 experiment and were replicated with similar results.

To provide further pharmacological evidence of non$\alpha 4 \beta 2$-mediated antiallodynia in SNI, we pretreated WT animals with a potent $\alpha 4 \beta 2$ antagonist, dihydro- $\beta$-erythroidine (DH $\beta E$ ), at a dose previously shown to block central effects of systemically administered nicotine $(33,34)$. After allodynia induction via SNI surgery, animals were injected subcutaneously with 1 $\mathrm{mg} / \mathrm{kg}$ DHßE 20 minutes before nicotine administration and tested for paw withdrawal thresholds (35). Animals pretreated with $\mathrm{DH} \beta \mathrm{E}$ and saline both exhibited robust nicotine-induced antiallodynia (Supplemental Figure 7H; saline, $0.630 \pm 0.171 \mathrm{~g}$; DH $\beta E, 0.702 \pm 0.131 \mathrm{~g})$.

Finally, to understand whether the clinical efficacy of ABT-594 may be mediated by $\alpha 6 \beta 4$, we injected a separate cohort of BARPKO mice with ABT-594 (0.3 mg/kg; i.p.). Importantly, ABT-594 alleviated mechanical allodynia in WT but not BARP-KO animals (Figure 6G), further supporting our model that BARP is crucial for $\alpha 6 \beta 4$ function and nicotinic agent-induced antiallodynia (WT SNI, $0.252 \pm 0.062 \mathrm{~g} ; \mathrm{WT}$ SNI + ABT-594, $1.102 \pm 0.341 \mathrm{~g} ;$ BARP-KO 
SNI, $0.210 \pm 0.051 \mathrm{~g} ;$ BARP-KO SNI + ABT-594, $0.479 \pm 0.232$ $\mathrm{g})$. Whereas previous studies found that the nonspecific nicotinic antagonist mecamylamine blocks ABT-594's antiallodynic effect (36), animals pretreated with DH $\beta E$ retained significant ABT-594 antiallodynia (Supplemental Figure 7I; saline, $2.568 \pm 0.422 \mathrm{~g}$; $\mathrm{DH} \beta \mathrm{E}, 2.059 \pm 0.292 \mathrm{~g}$ ), highlighting a non- $\alpha 4 \beta 2$ mechanism.

\section{Discussion}

The cholinergic system participates in numerous neurodegenerative and neuropsychiatric disorders. However, many nAChRs cannot be functionally expressed, which has impeded drug discovery. Genome-wide cDNA screening here identified accessory proteins required for activity of the previously intractable $\alpha 6 \beta 4$ receptor and unlock future high-throughput screening efforts. These findings expand the array of partner proteins that differentially control nAChRs. Whereas NACHO regulates most nAChRs, NACHO has no effect on $\alpha 6 \beta 4$. The $\alpha 6$-containing nAChRs themselves show selectivity, as NACHO, BARP, SULT2B1, and LAMP5 affect $\alpha 6 \beta 2 \beta 3$ (13), whereas $\alpha 6 \beta 4$ is regulated by BARP, IRE1 $\alpha$, and SULT2B1. These multiple layers of molecular control may allow precise regulation of $\mathrm{nAChRs}$ during specific developmental and physiological circumstances. Accordingly, $\alpha 6 \beta 2 \beta 3$ concentrates at presynaptic terminals of certain monoaminergic neurons, and this may be enabled by the lysosomal protein LAMP5, which displays a similar restricted distribution (37). On the other hand, LAMP5 is absent from DRG and does not regulate $\alpha 6 \beta 4$.

IRE1 $\alpha$ promotes $\alpha 6 \beta 4$ assembly via the UPR pathway. Previous studies in C. elegans identified a component of the ER membrane complex as critical for nAChR assembly and function (38). In mammals, nicotine-induced upregulation of nAChRs reduces the UPR including IRE1 $\alpha$, suggesting an important interplay (39, 40). Furthermore, a recent report identified a direct chaperone role for IRE1 $\alpha$ on $\mathrm{IP}_{3}$ receptors at mitochondria-associated membranes that did not require its kinase or RNase activities (41). In contrast, effects on $\alpha 6 \beta 4$ require IRE1 $\alpha$ 's canonical kinase and RNase functions. Several observations suggest that this new IRE1 $\alpha$ role on nAChRs is physiologically relevant. First, IRE1 $\alpha$ enhanced responses from a subset of $\mathrm{nAChR}$ combinations, but IRE1 $\alpha$ did not affect other channels. Second, ATF6 and PERK, 2 other arms of the UPR, failed to enhance assembly of nAChRs, suggesting that IRE1 $\alpha$ 's effects are not a generalized consequence of UPR activation. Third, knockout of endogenous IRE1 $\alpha$ decreased $\alpha 6 \beta 4$ binding and function. Finally, activating endogenous IRE1 $\alpha$ with Tm enhanced function of exogenous $\alpha 6 \beta 4$ and endogenous $\alpha 4 \beta 2$ in neurons.

Promotion of $\alpha 6 \beta 4$ assembly by IRE1 $\alpha$ fits conceptually with the latter's role as a sensor of the UPR, which promotes protein folding in the ER during cell stress (16). Indeed, effects on $\alpha 6 \beta 4$ involve the canonical IRE1 $\alpha$ pathway of autophosphorylation and splicing to form XBP1s. Once spliced, XBP1s translocates to the nucleus and upregulates chaperones such as Erdj4 and calnexin, which improves ER protein folding capacity (42-44). Accordingly, calnexin directly enhances assembly of muscle-type nAChRs (45). Precisely how $\mathrm{XBP} 1$ s promotes $\alpha 6 \beta 4$ assembly requires further study.

BARP promotes function of multiple nAChRs. BARP's robust enhancement of $\alpha 6 \beta 4$ function in HEK cells, oocytes, and neurons complements previous studies showing that BARP promotes function of $\alpha 6 \beta 2 \beta 3$ and $\alpha 3 \beta 2$ receptors (13). Whereas BARP regulates gating of $\alpha 6 \beta 2 \beta 3$ and $\alpha 3 \beta 2$ channels (13), it instead increases surface trafficking of $\alpha 6 \beta 4$. Thus, BARP enhances multiple $\alpha 6-$ containing nAChRs via distinct mechanisms. Yeast 2-hybrid screening originally identified BARP as a negative regulator of VGCCs. These studies found that 2 intracellular domains toward the BARP C-terminus bind to calcium channel $\beta$ subunits (14). However, the calcium channel-binding domains of BARP are not involved in regulation of $\alpha 6 \beta 4$. Instead, we find that a more membrane-proximal region between BARP amino acids 101 and 202 regulates $\alpha 6 \beta 4$. This suggests that BARP may function as a scaffold linking certain nAChRs with calcium channels. Such a model fits with experiments showing that cholinergic regulation of neurotransmitter release often involves calcium channel opening downstream of nAChRs $(46,47)$.

Targeting $\alpha 6 \beta 4$ receptors to treat neuropathic pain. Functional reconstitution of $\alpha 6 \beta 4$ receptors has important implications for neuropharmacology. Nicotine has long been recognized as an analgesic (48). Epibatidine is 200 times more potent than morphine in blocking nociception (49), but its side effects preclude clinical development (36). As several nAChR subtypes are expressed in DRG sensory neurons, and epibatidine is a pan-nicotinic agonist, the molecular target for its analgesia remains uncertain. $\alpha 4 \beta 2 \mathrm{nAChRs}$ have been classically thought to mediate nAChR-induced analgesia, yet years of research have yielded no approved therapeutics, suggesting involvement of a different receptor subtype (7). Recently, a genomics screen of DRG tissue from 25 inbred mouse strains identified the $\alpha 6 \mathrm{nAChR}$ subunit as the major phenotypic contributor to allodynia (8). Furthermore, knockout mouse studies showed that $\alpha 6$ but not $\alpha 4$ is required for peripheral and spinal nicotine-induced analgesia (8). The precise mechanisms through which nicotinic agents mediate analgesia are uncertain. Previous work suggested that $\alpha 6 \beta 4$ can produce analgesia via cross-inhibition of $\mathrm{P} 2 \mathrm{X} 2 / 3$ receptors on DRG neurons $(8,50)$. Activation of $\mathrm{nAChRs}$ may also promote analgesia via blunting of TrpV1-mediated currents in DRG nociceptors (51).

Here, we find that BARP-KO mice, which have impaired $\alpha 6 \beta 4$ surface expression in DRG, lack nicotine-mediated antiallodynia. Because BARP also promotes $\alpha 3 \beta 2$ and $\alpha 6 \beta 2 \beta 3$ function, we leveraged the fact that NACHO has no effect on $\alpha 6 \beta 4$ but promotes $\alpha 3 \beta 2$ and $\alpha 6 \beta 2 \beta 3$ assembly. We observed normal nicotineinduced allodynia in NACHO-KO mice, suggesting that the deficits observed in BARP-KO mice are mediated predominantly via $\alpha 6 \beta 4$ and not other nAChR subtypes. Supporting this, BARP KO did not alter surface expression of other nAChRs in DRG.

Phase II clinical studies found that ABT-594, a nicotinic agent related to epibatidine, was effective in treating diabetic neuropathic pain but had unacceptable side effects (29). The investigators hypothesized that activity at $\alpha 4 \beta 2$ was mediating analgesia, while $\alpha 3 \beta 4$ was predominantly responsible for the untoward side effects. As such, maximizing activity at $\alpha 4 \beta 2$ while minimizing $\alpha 3 \beta 4$ activity drove development of the follow-up compound, ABT-894. Unfortunately, ABT-894 was clinically ineffective (30). The study investigators hypothesized that "it is possible that ABT594 , which is a less selective $\alpha 4 \beta 2$ [neuronal nicotinic receptor (NNR)] agonist than ABT-894, elicits some of its effects via activity at non- $\alpha 4 \beta 2$ NNRs. If this is the case, the preferential affinity 
of ABT-894 for $\alpha 4 \beta 2$ NNRs may have been a disadvantage that resulted in a lack of analgesic efficacy" (30). Thus, the pharmacological difference between ABT-594 and -894 that accounts for their discrepant clinical efficacy is uncertain and likely involves non- $\alpha 4 \beta 2$ nAChRs.

Our ability to express functional $\alpha 6 \beta 4$ receptors showed that ABT-594 exhibits similar efficacy on $\alpha 4 \beta 2$ and $\alpha 6 \beta 4$. In contrast, ABT-894 exhibited only weak partial agonism on $\alpha 6 \beta 4$ as compared with $\alpha 4 \beta 2$ (Figure 6, D and E; Supplemental Table 1). We further show that the effective analgesics nicotine and epibatidine exhibit similar efficacies on $\alpha 4 \beta 2$ and $\alpha 6 \beta 4$. This unexpected pharmacology of $\alpha 6 \beta 4$ provides a likely explanation for the differential clinical efficacy of ABT- 594 versus ABT-894. That is, $\alpha 6 \beta 4$ activity contributed to ABT-594's clinical analgesia, yet ABT-894's diminished $\alpha 6 \beta 4$ efficacy may underpin the lack of its clinical efficacy. Supporting this model, ABT-594 exerts antiallodynic effects in WT mice but has no effect in BARP-KO mice, whose DRG have reduced $\alpha 6 \beta 4$ surface expression. Furthermore, pretreatment with the potent $\alpha 4 \beta 2$ antagonist DH $\beta E$ failed to block ABT-594induced antiallodynia, further supporting a non- $\alpha 4 \beta 2$ mechanism for alleviating neuropathic pain in the SNI model. ABT-594 alleviates thermal and inflammatory pain, suggesting that $\alpha 6 \beta 4$ agonists may be useful for a broad spectrum of pain conditions (36).

\section{Methods}

Cell culture and transfection. Expression plasmids for the following genes were used in this study (all human, GenBank accession numbers): CHRNA6 (NM_004198), CHRNA4 (NM_000744.6), CHRNA3 (NM_000743.5), CHRNA7 (NM_000746.5), CHRNB2 (NM_000748.2), CHRNB3 (NM_000749.5), CHRNB4 (NM_000750), BARP (XM_017026555), ERN1 (IRE1 $)$ ), PERK (NM_001313915.1), ATF6 (NM_007348.4), NACHO (NM_021637.2), SULT2B1 (NM_177973), LAMP5 (NM_012261.4), 5HT3A (NM_213621.4), GLUA1 (NM_000827.4), GABBR1 (NM_021903.2), and GABBR2 (NM_005458.8).

HEK293T cells were initially purchased from ATCC and cultured in DMEM high-glucose medium supplemented with 10\% FBS and 1 mM sodium pyruvate. Cells were transfected at $80 \%-90 \%$ confluence more than 4 hours after plating with FuGENE 6 or ViaFect Transfection Reagent (Promega) and incubated at $37^{\circ} \mathrm{C}$. To promote surface expression, cells were switched to $30^{\circ} \mathrm{C}$ after 24 hours. All assays unless otherwise noted were performed 48-72 hours after transfection. For cDNA library screening, expression-ready cDNA plasmids from a Broad Institute library were cotransfected in 384-well plates with $\alpha 6 \beta 4$ subunits in a 1:1:1 ratio.

For neuronal FLIPR and epibatidine experiments, rat cortical E18 neurons were purchased from BrainBits, dissociated with papain, and cultured in NbActiv media (BrainBits) at $37^{\circ} \mathrm{C}$. Cultures were transduced with lentiviral particles expressing $\alpha 6$ and $\beta 4$ (Vigene Biosciences) at DIV 7 and assayed at DIV 13. Cultures were pretreated with compounds for indicated time periods before assay.

For DRG neuron staining, DRGs from adult WT or $B A R P^{-/-}$mice were dissected in cold L-15 media supplemented with GlutaMAX (Thermo Fisher Scientific). DRGs were dissociated with collagenase/ protease IV solution for 60 minutes. DRGs were triturated with a fire-polished glass pipette, resuspended in L-15 media, and filtered with a cell strainer. Cells were then pipetted on top of a $60 \% / 30 \%$ Percoll gradient and centrifuged at $800 \mathrm{~g}$ for 20 minutes at $4^{\circ} \mathrm{C}$. After centrifugation, the top layer was discarded, and neurons were collected and spun down at $200 \mathrm{~g}$ for 10 minutes. The supernatant was aspirated, and cells were resuspended in Neurobasal A supplemented with B-27, GlutaMAX, and $25 \mathrm{ng} / \mathrm{mL}$ nerve growth factor. Neurons were then plated on glass coverslips coated with poly-D-lysine (PDL) and laminin. Cells were transduced 1 hour after plating and assayed 5 days later.

SNI and tactile threshold testing. For the SNI procedure, adult male mice (23-33 g) were deeply anesthetized with isoflurane and SNI surgery was performed as described previously (31). Briefly, the sural and common peroneal branches of the left sciatic nerve were ligated, leaving the tibial branch intact. Only mice that did not exhibit motor dysfunction (e.g., paw dragging) were used.

For tactile threshold testing, following a 30-minute acclimation on the von Frey stand, preinjury tactile thresholds were measured from the left hind paw and recorded before SNI surgery. Fifty-percent tactile paw withdrawal thresholds (PWTs) were measured from the left paw and recorded using calibrated von Frey filaments applied perpendicularly with sufficient force to bend slightly and held for 2-3 seconds against the plantar surface. Paw flinching during or immediately following removal of the stimulus was scored as positive. PWT was tested at preinjury levels and at $1,3,7,14$, and 21 days (21 days deemed SNI) following surgery. At 29 days following surgery, mice were i.p. injected with vehicle, $1.0 \mathrm{mg} / \mathrm{kg}(-)$-nicotine (MilliporeSigma), or $0.3 \mathrm{mg} / \mathrm{kg} \mathrm{ABT-594}$ (Tocris) and again tested at either 10 minutes (nicotine) or 1 hour (ABT-594) after injection. For DH $\beta$ E pretreatment experiments, $\mathrm{DH} \beta \mathrm{E}$ was dissolved in saline and administered subcutaneously at a final concentration of $1 \mathrm{mg} / \mathrm{kg} 20$ minutes before nicotine or ABT-594 injection. Experimenters were blinded to genotype throughout the study.

Molecular biology. IRE1 $\alpha$ mutants (K907A, RNAmut; K599A, KINmut; I642G, CONDmut) were generated via site-directed mutagenesis and confirmed by sequencing. BARP101 and BAPR202 truncation mutants were generated by inserting a premature stop codon at amino acid 102 and 203, respectively.

The $\alpha 4 \mathrm{~N} / 6$ chimera consisted of the first 242 amino acids corresponding to the $\mathrm{N}$-terminal extracellular region of $\alpha 4$ fused to the transmembrane and intracellular regions of $\alpha 6$ (amino acids 240-495). Conversely, the $\alpha 6 \mathrm{~N} / 4$ chimera consisted of the first 239 amino acids of $\alpha 6$ fused to the transmembrane and intracellular regions of $\alpha 4$ (amino acids 243-628). Loop chimeras substituted amino acids 331-600 for $\alpha 4$ and 328-465 for $\alpha 6$. All chimeras were confirmed by sequencing.

To generate $I R E 1 \alpha-\mathrm{KO}$ cell lines, a guide RNA targeting exon 2 of the IRE1 $\alpha$ gene was cotransfected into HEK293T cells with Cas9 enzyme (IDT Technologies) and single-stranded oligodeoxynucleotide (ssODN) for homology-directed repair using TransIT-X2 transfection reagent (Mirus, MIR6003). Serine at position 36 was mutated to a stop codon. Single clones were isolated, expanded, sequenced, and confirmed for hetero- or homozygosity with a Zero Blunt TOPO PCR Cloning Kit (Thermo Fisher Scientific, K280020SC). ssODN sequence: CAGATTTTTGGAAGTACCAGCACAGTGACGCTTCCTGAAACCTTGTTGTTTGTGTAAACGCTGGATGGAAGTTTGCATGCTGTCAGCAAGAGGACAGGCTCAATCAAATGG.

For XBP1s RT-PCR experiments, HEK293T cells were seeded in 6-well plates and treated with vehicle or Tm for the indicated time points. Cells were then collected and RNA isolated using an RNeasy Mini Kit (QIAGEN, 74104). cDNA was synthesized from total RNA 
with a SuperScript IV First-Strand Synthesis System (Thermo Fisher Scientific, 18091050) according to the manufacturer's instructions. The following primers were used to amplify XBP1 cDNA with PCR (25): XBP1sense, TTACGAGAGAAAACTCATGGC; antisense, GGGTCCAAGTTGTCCAGAATGC.

FLIPR assays. HEK293T cells were seeded at 15,000/well in 384-well glass-bottomed PDL-coated plates (Corning). At the time of assay, cells were washed once with FLIPR buffer (HyClone HEPES-buffered saline [GE Life Sciences] with [in mM] $149 \mathrm{NaCl}$, $4 \mathrm{KCl}, 10$ HEPES, 5 glucose, $2 \mathrm{CaCl}_{2}, 1 \mathrm{MgCl}_{2} ; 300 \mathrm{mOsm}$ and $\mathrm{pH}$ 7.4) and loaded for 1 hour at room temperature with Calcium 5 dye (Molecular Devices). After dye loading, excess dye was removed, plates were washed once with FLIPR buffer, and then placed in a FLIPR Tetra (Molecular Devices) machine. Compound-induced $\mathrm{Ca}^{2+}$ responses were monitored and measured for 5 minutes with Screenworks software (Molecular Devices). For $\alpha 6 \beta 2 \beta 3^{*}$ responses, $\alpha 6 / 3$ chimera and $\beta 3$ mutant were used to enable larger responses (13). For $\alpha 7$ responses, cells were first incubated with the positive allosteric modulator PNU-120596 (5 $\mu \mathrm{M})$. Unless otherwise noted, all FLIPR transfections were carried out at a 3:1:5 ratio ( $\alpha 6 / \beta 4 /$ chaperone). XBP1s transfections were carried out at a ratio of 3:1:0.1 ( $\alpha 6 / \beta 4 /$ $\mathrm{XBP} 1 \mathrm{~s})$. For neuronal assays, the FLIPR protocol was identical except that $3 \mathrm{mM} \mathrm{CaCl}_{2}$ was used in FLIPR buffer. To isolate $\alpha 6 \beta 4$ responses from endogenously expressed $\alpha 4 \beta 2$, neurons were incubated for 5 minutes with $25 \mu \mathrm{M}$ DH $\beta E$ before stimulation with nicotine.

Immunofluorescent surface staining. HEK293T cells were seeded at 10,000/well in 384-well glass-bottomed PDL-coated plates (Corning). All cell transfections included EGFP to label cytoplasm of transfected cells. For staining, cells were incubated for 1 hour with fluorescent-conjugated antibody against HA or V5 tag (Dylight HA-650 [26183-D650], Alexa Fluor V5-555 [37-7500-A555], Invitrogen) at $30^{\circ} \mathrm{C}$. Cells were washed with PBS and then fixed for 45 minutes ar room temperwature with $4 \%$ paraformaldehyde. After washing, nuclei were stained with NucBlue solution (Invitrogen). Plates were imaged using the Opera Phenix confocal instrument (PerkinElmer) at $\times 20$ magnification. For image analysis, Columbus automated software was used (PerkinElmer). Briefly, EGFP labeling was used for automatic cell detection. Then, average fluorescence intensity was measured in a ring region surrounding the cell, but not including the cytoplasm, which represented surface staining.

For DRG neuron staining, cells were live stained with the same anti-V5 primary antibody as described above. Cells were additionally costained with $\beta-3$ tubulin (Tuj1) conjugated to Alexa Fluor 488 (eBioscience, 53-4510-82) to label cell bodies and processes. For cytoplasmic staining, cells were fixed then permeabilized for 20 minutes at room temperature with $0.3 \%$ Triton $\mathrm{X}-100$ in PBS. Neurons were imaged at $\times 40$ magnification with a confocal microscope (Zeiss).

$\left[{ }^{3} \mathrm{H}\right]$ epibatidine binding. HEK293T cells or rat cortical E18 neurons were collected with PBS, spun down, and then resuspended in $50 \mathrm{mM}$ ice-cold Trizma buffer (MilliporeSigma). Cells were homogenized for 30 seconds at max speed with a T-25 Ultra-Turrax homogenizer (Ika) and concentrations measured using the Pierce BCA assay (Thermo Fisher Scientific). Samples were run in 16 replicates and incubated with $10 \mathrm{nM}\left[{ }^{3} \mathrm{H}\right]$ epibatidine (PerkinElmer) for 2-3 hours at room temperature. In half of the samples, $10 \mu \mathrm{M}$ unlabeled epibatidine (Tocris) was coapplied to determine nonspecific binding. Samples were filtered and bound to polyethylenimine-treated 96-well GF/B filter plates (PerkinElmer). Plates were washed with cold Trizma buffer and then desiccated for 20 minutes at $65^{\circ} \mathrm{C}$. Each well received $60 \mu \mathrm{L}$ of MicroScient-0, and then plates were read with a TopCount NXT scintillation counter (PerkinElmer).

Protein biochemistry. For immunoblotting, cells were collected into RIPA buffer (Thermo Fisher Scientific) with Halt protease and phosphatase inhibitor cocktail (Thermo Fisher Scientific). Cells were sonicated, spun down at $14,000 \mathrm{~g}$ for 30 minutes at $4^{\circ} \mathrm{C}$, and the supernatant was taken for further analysis. Protein concentrations were measured using the Pierce BCA assay. Protein lysates were denatured at $55^{\circ} \mathrm{C}$ for 30 minutes, proteins were resolved by SDS-PAGE, and blots were incubated overnight at $4^{\circ} \mathrm{C}$ with primary antibodies (anti- $\beta$ actin, Thermo Fisher Scientific [PA516914]; anti-IRE1 $\alpha$, Cell Signaling Technology [3294S]; anti-HA, Thermo Fisher Scientific [26183]; anti-V5, Thermo Fisher Scientific [PA1993]; and anti-BARP [ref. 13]). Blots were then incubated for 1 hour at room temperature in the dark with infrared-conjugated secondary antibodies and imaged using the Odyssey system (Licor).

For coimmunoprecipitation, cells were solubilized 2 hours at $4^{\circ} \mathrm{C}$ on a shaker in buffer containing $100 \mathrm{mM} \mathrm{NaCl}, 1 \% n$ dodecyl-D-maltoside, and $1 \mathrm{mM}$ iodoacetamide. Samples were spun at $20,000 \mathrm{~g}$ at $4^{\circ} \mathrm{C}$ for 45 minutes. These lysates were incubated with $3 \mu \mathrm{g}$ of immunoprecipitation antibody on a shaker overnight at $4^{\circ} \mathrm{C}$. Protein-antibody complexes were captured with Protein A Dynabeads (Thermo Fisher Scientific). After washing, bound proteins were eluted by heating to $55^{\circ} \mathrm{C}$ for 30 minutes and were analyzed by immunoblotting.

Oocyte recordings. cRNAs were obtained via in vitro transcription with an mMessage mMACHINE T7 Transcription Kit (Thermo Fisher Scientific, AM1344). Stage 4/5 oocytes were purchased from EcoCyte Biosciences and injected with 60 ng total cRNA at a ratio of 1:1:2 $\alpha 6 / \beta 4 /$ chaperone. After injection, oocytes were placed in Modified Barth's Saline (MilliporeSigma) supplemented with gentamycin and incubated at $18^{\circ} \mathrm{C}$. Recordings were made 3-5 days after cRNA injection and stimulated with $250 \mu \mathrm{M}$ ACh for 2 seconds. Oocytes were recorded using the 2-electrode voltage-clamp method and held at -60 $\mathrm{mV}$. Currents were acquired using a HEKA amplifier with FitMaster software (HEKA Elektronik). Data were sampled at $5 \mathrm{kHz}$ and filtered with a 4 -pole Bessel filter at $1 \mathrm{kHz}$.

Electrophysiology. HEK293T cells in 6-well plates were cotransfected with $\alpha 6 \beta 4$, chaperones, and EGFP, and 24 hours before recording were replated on 12-mm glass coverslips. For recordings, coverslips were placed in an extracellular bath composed of (in $\mathrm{mM}$ ) $149 \mathrm{NaCl}, 4 \mathrm{KCl}, 10$ HEPES, 5 glucose, $2 \mathrm{CaCl}_{2}$, and $1 \mathrm{MgCl}_{2}$. Borosilicate glass (World Precision Instruments) pipettes were pulled to obtain final resistances of 3-5 M $\Omega$ with a PC-10 micropipette puller (Narshige). Internal solution comprised (in mM) $145 \mathrm{CsCl}, 2.5 \mathrm{NaCl}$, 10 HEPES, 4 MgATP, 1 EGTA; pH was adjusted to 7.4 with $\mathrm{CsOH}$; osmolality was adjusted to $300 \mathrm{mOsm} / \mathrm{kg}$ with sucrose. After wholecell recording from $\mathrm{EGFP}^{+}$cells was achieved, cells were carefully lifted from the coverslip and moved toward a separate pipette with agonist. To record agonist-evoked responses, ACh was rapidly applied to cells held at $-60 \mathrm{mV}$ using a MXPZT piezoelectric actuator system (Siskiyou). Series resistances (3-15 M $\Omega$ ) were compensated by at least $80 \%$. All recordings were carried out with an Axopatch $200 B$ amplifier (Molecular Devices). Signals were sampled at $25 \mathrm{kHz}$ and low-pass filtered at $10 \mathrm{kHz}$. Data were acquired and analyzed 
using pClamp10 software (Molecular Devices). Steady state/peak ratio was calculated as the amplitude of steady-state current during constant ACh application divided by the peak current.

Statistics. Results are represented as mean \pm SEM unless otherwise noted. For data sets with 2 groups, an unpaired, 2-tailed Student's $t$ test was used to test for significance. For 3 or more groups, 1-way ANOVA was used. To adjust for multiple comparisons: when comparing multiple groups to a control group, Dunnett's post hoc test was used. If differences between all groups to each other were tested, then Tukey's post hoc test was used. Significance levels were set at ${ }^{*} P<0.05,{ }^{* *} P<0.01$, ${ }^{* *} P<0.001$ unless otherwise noted in figure legends. The D'Agostino and Pearson omnibus test with an $\alpha$ value of 0.05 was used to test for normality. $\mathrm{EC}_{50}$ values and concentration-response curves were calculated using a variable slope, 4-parameter nonlinear regression model: $y=$ bottom $+\left(x^{\text {Hill slope }}\right) \times($ top - bottom $) /\left(x^{\text {Hill slope }}+\mathrm{EC}_{50}{ }^{\text {Hill slope }}\right)$. All statistics and graphing were carried out with Prism (GraphPad).

Study approval. BARP-KO and NACHO-KO mice were previously described $(12,13)$. All procedures were performed in accordance with Janssen (La Jolla) IACUC guidelines.

\section{Author contributions}

DK designed and conducted experiments, analyzed the data, and prepared the manuscript. SG helped conduct experiments. WAE performed mouse behavioral studies. GBD and JAM conducted HEK293T electrophysiology experiments. JL and ADW helped with pharmacological experiments. AB supervised behavioral studies. DSB designed experiments, supervised the project, and prepared the manuscript. All authors provided input for writing manuscript. Co-authorship was assigned by time of initial involvement.

\section{Acknowledgments}

The authors thank Ondrej Libiger for statistical advice, Bridget Kohlnofer for advice on CRISPR knockout generation, and Mike Morton for technical assistance with cell sorting.

Address correspondence to: David S. Bredt, Janssen Pharmaceutical Companies of Johnson \& Johnson, 3210 Merryfield Row, San Diego, California, USA. Phone: 858.320.3374; Email: dbredt@its.jnj.com.
1. Role LW, Berg DK. Nicotinic receptors in the development and modulation of CNS synapses. Neuron. 1996;16(6):1077-1085.

2. Gotti C, Clementi F. Neuronal nicotinic receptors: from structure to pathology. Prog Neurobiol. 2004;74(6):363-396.

3. Lindstrom J. Nicotinic acetylcholine receptors in health and disease. Mol Neurobiol. 1997;15(2):193-222.

4. Hogg RC, Raggenbass M, Bertrand D. Nicotinic acetylcholine receptors: from structure to brain function. Rev Physiol Biochem Pharmacol. 2003;147:1-46.

5. Le Novère N, Corringer PJ, Changeux JP. The diversity of subunit composition in nAChRs: evolutionary origins, physiologic and pharmacologic consequences. J Neurobiol. 2002;53(4):447-456.

6. Picciotto MR. Nicotine as a modulator of behavior: beyond the inverted U. Trends Pharmacol Sci. 2003;24(9):493-499.

7. Hone AJ, McIntosh JM. Nicotinic acetylcholine receptors in neuropathic and inflammatory pain. FEBS Lett. 2018;592(7):1045-1062.

8 . Wieskopf JS, et al. The nicotinic $\alpha 6$ subunit gene determines variability in chronic pain sensitivity via cross-inhibition of $\mathrm{P} 2 \mathrm{X} 2 / 3$ receptors. $\mathrm{Sci}$ Transl Med. 2015;7(287):287ra72.

9. Wang J, Kuryatov A, Lindstrom J. Expression of cloned $\alpha 6^{*}$ nicotinic acetylcholine receptors. Neuropharmacology. 2015;96(pt B):194-204.

10. Letchworth SR, Whiteaker P. Progress and challenges in the study of $\alpha 6$-containing nicotinic acetylcholine receptors. Biochem Pharmacol. 2011;82(8):862-872.

11. Gu S, et al. Brain $\alpha 7$ nicotinic acetylcholine receptor assembly requires NACHO. Neuron. 2016;89(5):948-955.

12. Matta JA, et al. NACHO mediates nicotinic acetylcholine receptor function throughout the brain. Cell Rep. 2017;19(4):688-696.

13. Gu S, Matta JA, Davini WB, Dawe GB, Lord B, Bredt DS. $\alpha 6$-Containing nicotinic acetylcholine receptor reconstitution involves mechanisti- cally distinct accessory components. Cell Rep. 2019;26(4):866-874.e3.

14. Béguin $P$, et al. BARP suppresses voltage-gated calcium channel activity and $\mathrm{Ca}^{2+}$-evoked exocytosis. J Cell Biol. 2014;205(2):233-249.

15. Chen Y, Brandizzi F. IRE1: ER stress sensor and cell fate executor. Trends Cell Biol. 2013;23(11):547-555.

16. Walter P, Ron D. The unfolded protein response: from stress pathway to homeostatic regulation. Science. 2011;334(6059):1081-1086.

17. Kuryatov A, Olale F, Cooper J, Choi C, Lindstrom J. Human alpha6 AChR subtypes: subunit composition, assembly, and pharmacological responses. Neuropharmacology. 2000;39(13):2570-2590.

18. Papke RL, et al. Extending the analysis of nicotinic receptor antagonists with the study of alpha6 nicotinic receptor subunit chimeras. Neuropharmacology. 2008;54(8):1189-1200.

19. Dash B, Bhakta M, Chang Y, Lukas RJ. Identification of $\mathrm{N}$-terminal extracellular domain determinants in nicotinic acetylcholine receptor (nAChR) $\alpha 6$ subunits that influence effects of wild-type or mutant $\beta 3$ subunits on function of $\alpha 6 \beta 2^{*}$ - or $\alpha 6 \beta 4^{*}$-nAChR. J Biol Chem. 2011;286(44):37976-37989.

20. Kouvatsos N, Giastas P, Chroni-Tzartou D, Poulopoulou C, Tzartos SJ. Crystal structure of a human neuronal nAChR extracellular domain in pentameric assembly: Ligand-bound $\alpha 2$ homopentamer. Proc Natl Acad Sci U S A. 2016;113(34):9635-9640.

21. Han D, et al. IRE1alpha kinase activation modes control alternate endoribonuclease outputs to determine divergent cell fates. Cell. 2009;138(3):562-575.

22. Maly DJ, Papa FR. Druggable sensors of the unfolded protein response. Nat Chem Biol. 2014;10(11):892-901.

23. Papa FR, Zhang C, Shokat K, Walter P. Bypassing a kinase activity with an ATP-competitive drug. Science. 2003;302(5650):1533-1537.

24. Jiang $\mathrm{H}$, et al. Unfolded protein response induc- ers tunicamycin and dithiothreitol promote myeloma cell differentiation mediated by XBP-1. Clin Exp Med. 2015;15(1):85-96.

25. Lin JH, et al. IRE1 signaling affects cell fate during the unfolded protein response. Science. 2007;318(5852):944-949.

26. Ray P, et al. Comparative transcriptome profiling of the human and mouse dorsal root ganglia: an RNA-seq-based resource for pain and sensory neuroscience research. Pain. 2018;159(7):1325-1345.

27. Hone AJ, Meyer EL, McIntyre M, McIntosh JM. Nicotinic acetylcholine receptors in dorsal root ganglion neurons include the $\alpha 6 \beta 4^{*}$ subtype. FASEB J. 2012;26(2):917-926.

28 . Moser N, et al. Evaluating the suitability of nicotinic acetylcholine receptor antibodies for standard immunodetection procedures. J Neurochem. 2007;102(2):479-492.

29. Rowbotham MC, Duan WR, Thomas J, Nothaft W, Backonja MM. A randomized, double-blind, placebo-controlled trial evaluating the efficacy and safety of ABT-594 in patients with diabetic peripheral neuropathic pain. Pain. 2009;146(3):245-252.

30. Rowbotham MC, et al. Efficacy and safety of the $\alpha 4 \beta 2$ neuronal nicotinic receptor agonist ABT894 in patients with diabetic peripheral neuropathic pain. Pain. 2012;153(4):862-868.

31. Shields SD, Eckert WA, Basbaum AI. Spared nerve injury model of neuropathic pain in the mouse: a behavioral and anatomic analysis. J Pain. 2003;4(8):465-470.

32. Kyte SL, et al. Nicotine prevents and reverses paclitaxel-induced mechanical allodynia in a mouse model of CIPN. J Pharmacol Exp Ther. 2018;364(1):110-119.

33. Davis JA, Gould TJ. The effects of DHBE and MLA on nicotine-induced enhancement of contextual fear conditioning in C57BL/6 mice. Psychopharmacology (Berl). 2006;184(3-4):345-352.

34. Li S, et al. Nicotine inhibits rapamycin-induced pain through activating mTORC1/S6K/IRS-1related feedback inhibition loop. Brain Res Bull. 
2019;149:75-85.

35. Damaj MI, Welch SP, Martin BR. In vivo pharmacological effects of dihydro-beta-erythroidine, a nicotinic antagonist, in mice. Psychopharmacology (Berl). 1995;117(1):67-73.

36. Bannon AW, et al. Broad-spectrum, non-opioid analgesic activity by selective modulation of neuronal nicotinic acetylcholine receptors. Science. 1998;279(5347):77-81.

37. Tiveron MC, et al. LAMP5 fine-tunes GABAergic synaptic transmission in defined circuits of the mouse brain. PLoS One. 2016;11(6):e0157052.

38. Richard M, Boulin T, Robert VJ, Richmond JE, Bessereau JL. Biosynthesis of ionotropic acetylcholine receptors requires the evolutionarily conserved ER membrane complex. Proc Natl Acad Sci U S A. 2013;110(11):E1055-E1063.

39. Srinivasan R, et al. Pharmacological chaperoning of nicotinic acetylcholine receptors reduces the endoplasmic reticulum stress response. $\mathrm{Mol}$ Pharmacol. 2012;81(6):759-769.

40. Srinivasan R, et al. Smoking-relevant nicotine concentration attenuates the unfolded protein response in dopaminergic neurons. J Neurosci.
2016;36(1):65-79.

41. Carreras-Sureda A, et al. Non-canonical function of IRE $1 \alpha$ determines mitochondria-associated endoplasmic reticulum composition to control calcium transfer and bioenergetics. Nat Cell Biol. 2019;21(6):755-767.

42. Lee AH, Iwakoshi NN, Glimcher LH. XBP-1 regulates a subset of endoplasmic reticulum resident chaperone genes in the unfolded protein response. Mol Cell Biol. 2003;23(21):7448-7459.

43. Rutkowski DT, et al. The role of p58IPK in protecting the stressed endoplasmic reticulum. $\mathrm{Mol}$ Biol Cell. 2007;18(9):3681-3691.

44. Shoulders MD, et al. Stress-independent activation of XBP1s and/or ATF6 reveals three functionally diverse ER proteostasis environments. Cell Rep. 2013;3(4):1279-1292.

45. Chang W, Gelman MS, Prives JM. Calnexindependent enhancement of nicotinic acetylcholine receptor assembly and surface expression. J Biol Chem. 1997;272(46):28925-28932.

46. McGehee DS, Heath MJ, Gelber S, Devay P, Role LW. Nicotine enhancement of fast excitatory synaptic transmission in CNS by presynaptic receptors. Science. 1995;269(5231):1692-1696.

47. Kulak JM, McIntosh JM, Yoshikami D, Olivera BM. Nicotine-evoked transmitter release from synaptosomes: functional association of specific presynaptic acetylcholine receptors and voltage-gated calcium channels. J Neurochem. 2001;77(6):1581-1589.

48. Mattila MJ, Ahtee L, Saarnivaara L. The analgesic and sedative effects of nicotine in white mice, rabbits and golden hamsters. Ann Med Exp Biol Fenn. 1968;46(1):78-84.

49. Daly JW, Garraffo HM, Spande TF, Decker MW, Sullivan JP, Williams M. Alkaloids from frog skin: the discovery of epibatidine and the potential for developing novel non-opioid analgesics. Nat Prod Rep. 2000;17(2):131-135.

50. Limapichat W, Dougherty DA, Lester HA. Subtype-specific mechanisms for functional interaction between $\alpha 6 \beta 4^{*}$ nicotinic acetylcholine receptors and $\mathrm{P} 2 \mathrm{X}$ receptors. Mol Pharmacol. 2014;86(3):263-274.

51. Fucile S, Sucapane A, Eusebi F. $\mathrm{Ca}^{2+}$ permeability of nicotinic acetylcholine receptors from rat dorsal root ganglion neurones. J Physiol (Lond). 2005;565(pt 1):219-228. 